ARTICLE

https://doi.org/10.1038/s41467-020-14323-y

\title{
A MAFG-IncRNA axis links systemic nutrient abundance to hepatic glucose metabolism
}

Marta Pradas-Juni et al."

Obesity and type 2 diabetes mellitus are global emergencies and long noncoding RNAs (IncRNAs) are regulatory transcripts with elusive functions in metabolism. Here we show that a high fraction of IncRNAs, but not protein-coding mRNAs, are repressed during diet-induced obesity (DIO) and refeeding, whilst nutrient deprivation induced IncRNAs in mouse liver. Similarly, IncRNAs are lost in diabetic humans. LncRNA promoter analyses, global cistrome and gain-of-function analyses confirm that increased MAFG signaling during DIO curbs IncRNA expression. Silencing Mafg in mouse hepatocytes and obese mice elicits a fasting-like gene expression profile, improves glucose metabolism, de-represses IncRNAs and impairs mammalian target of rapamycin (mTOR) activation. We find that obesity-repressed LinclRS2 is controlled by MAFG and observe that genetic and RNAi-mediated LincIRS2 loss causes elevated blood glucose, insulin resistance and aberrant glucose output in lean mice. Taken together, we identify a MAFG-IncRNA axis controlling hepatic glucose metabolism in health and metabolic disease.

\#A full list of authors and their affiliations appears at the end of the paper. 
C ellular and organism-level energy homeostasis and nutrient partitioning are instrumental for survival. In higher organisms, multi-organ systems evolved to react to shifts in energy supply by storing (anabolic) or metabolizing (catabolic) nutrients, for instance by conversion of simple sugars to storage macromolecules like glycogen or by glucose catabolism, according to caloric demands ${ }^{1}$. A key process for mounting appropriate responses to altered nutrient availabilities are abundance, localization, and nutrient-induced activation of transcriptional networks that couple energy states to appropriate changes in gene expression $^{2}$. These ancient molecular circuits ensured survival of animals during (historically frequent) food shortages. In contrast, the exposure to constant calorie overload, coupled with sedentary lifestyles, results in excess storage of nutrients, and concomitantly, obesity and obesity-associated maladies. Globally, two billion individuals are considered overweight or obese and exhibit elevated risks of developing severe comorbidities such as cardiovascular disease ${ }^{3}$, artherosclerosis ${ }^{4}$, type 2 diabetes mellitus (T2D), liver steatosis, or nonalcoholic fatty liver disease (NAFLD) and nonalcoholic steatohepatitis $(\mathrm{NASH})^{5}$. Therefore, understanding how nutrient-sensitive signaling networks are controlled during conditions of energy surplus, and whether this can be prevented in obesity, will be instrumental in designing effective therapies in the future.

Remarkable for living in seemingly postgenomic times, we currently witness a paradigm shift in understanding our genomes and the information contained therein: multinational sequencing efforts like $\mathrm{ENCODE}^{6}$, $\mathrm{FANTOM}^{7}$, or $\mathrm{NONCODE}^{8}$, together with increasing RNA-Sequencing (RNA-Seq) capabilities and reduced costs, led to the intriguing discovery that, whereas only $1-2 \%$ of genomic information encodes protein-coding mRNAs, more than $70 \%$ of DNA is transcribed across developmental space and time $\mathrm{e}^{9,10}$. This led to the identification of thousands of microRNAs, and more recently, long noncoding RNAs (lncRNAs) $)^{11,12}$ in mice and humans ${ }^{13}$. The identification of these many lncRNAs in silico and ascribing a specific function to each has been challenging ${ }^{14}$. Functions identified to date include microRNA scavenging ${ }^{15}$, chromatin sequestration ${ }^{16}, 3 \mathrm{D}$ genome $\operatorname{organization}^{17}$, chromatin modifier recruitment ${ }^{18}$, lncRNA-DNA triplex formation ${ }^{19}$, and small-molecule protein complex formation $^{20}$ to control mRNA translation ${ }^{15}$ and RNA-RNA crosstalk ${ }^{21}$.

Investigators have only begun to ascribe specific in vivo functions for lncRNAs in cellular energy homeostasis and/or metaboregulatory signaling circuits. Such functions of lncRNAs include control of hepatic cholesterol biosynthesis ${ }^{22}$, de novo lipogenesis and systemic lipid homeostasis ${ }^{23,24}$, beta-oxidation ${ }^{25}$, glucose homeostasis ${ }^{26,27}$, and regulation of insulin sensitivity in the liver ${ }^{27}$. Understanding the molecular details of these regulatory processes may lead to innovative approaches where repression of specific lncRNAs may prove useful in the treatment of metabolic diseases.

By performing RNA-Seq in mouse models for dietary and genetic obesity, T2D, and livers from diabetic human patients, we find that nutrient excess and refeeding (RF) favors lncRNA repression, whereas fasting induces lncRNA expression. Intriguingly, protein-coding mRNAs are not affected accordingly. In silico analyses of lncRNA and mRNA promoters, and integration of MAFG ChIP-Seq with MAFG gain-of-function RNASeq data confirm that elevated MAFG signaling in obesity and $\mathrm{RF}$ is transcriptionally linked to lncRNA repression. In vitro and in vivo loss of MAFG in hepatocytes controls glucose production, improves glucose metabolism during obesity, and induces lncRNAs, whereas MAFG gain of function represses hepatic lncRNAs. Intriguingly, MAFG loss prevents insulinevoked activation of mTORC1 signaling, thus presumably interfering with protein translation in hepatocytes. We further observe that obesity-repressed LincIRS2 is negatively controlled by MAFG and CRISPR-Cas9-mediated knockout, or antisensemediated RNA interference of LincIRS2 causes hyperglycemia, insulin resistance, likely caused by alterations in glucogenic gene expression in lean mice.

\section{Results}

Nutrient states elicit opposing effects on mRNA and lncRNAs. To identify lncRNAs that are implicated in the development of liver disease pathologies in diet-induced obesity (DIO), for instance insulin resistance, steatosis, and liver inflammation, 6-week-old C57BL/6N mice were fed a high-fat diet (HFD) or control diet (CD). After 30 weeks, hepatic RNA was isolated and total RNASequencing (RNA-Seq) performed. This approach identified 583 mRNAs and 50 lncRNAs that were significantly $(p$ value $(\mathrm{pV})<0.05$ by Student's $t$ test, false-discovery rate $<0.05$, and CuffDiff function significant $=$ "yes" for Benjamini-Hochberg correction for multiple testing) altered after HFD feeding (Fig. 1a, Supplementary Data 1). Performing Ingenuity Pathway Analysis (IPA) confirmed activation of transcription factor (TF) networks and signaling pathways known to be induced in the liver under anabolic/nutrient-rich conditions. These included insulin receptor (IR), Forkhead Box O1 (FOXO1), and Sterol Regulatory Element Binding Transcription Factor 1C (SREBP1C) pathways (Supplementary Fig. 1a-c). We interpreted these transcriptional changes as a reflection of chronic nutrient exposure that in turn triggers anabolic TF pathways in the liver.

When performing differential gene expression (DGE) analysis independently for protein-coding or lncRNA transcripts (gene biotypes from Ensembl Biomart ${ }^{28}$ ), we observed a significant cumulative downregulation of lncRNAs as compared with protein-coding mRNAs (Fig. 1c) in obese livers using nonparametric Kolmogorov-Smirnov tests. Prompted by this difference in mRNA vs. IncRNA regulation after HFD feeding, we repeated the analysis after physiological nutrient changes outside of obesity and metabolic disease. Comparing transcriptomes from ad libitum (AL)-fed C57BL/6N (wild-type) mice compared with mice fasted for $16 \mathrm{~h}$ (FA, Fig. 1b, Supplementary Data 2), we observed 1165 mRNAs and 92 lncRNAs differentially regulated. In contrast to lncRNA repression in obesity, lncRNAs were induced after fasting when compared with mRNAs (Fig. 1d). Short-term (6h) RF altered 587 mRNAs and 59 lncRNAs (Supplementary Data 3) with similar trends for induction of lncRNAs as compared with mRNAs (Supplementary Fig. 1d). We performed RNA-Seq in BKS.Cg-Dock ${ }^{7 m+/+}$; $L e p R^{d b} / J$ (termed $L e p R^{d b}$ ) mice, a genetic model of obesity and T2D, and observed 959 mRNAs and 83 lncRNAs significantly changed (Supplementary Data 4). Again, hepatic lncRNAs were decreased compared with mRNAs (Supplementary Fig. 1e) when we compared $L e p R^{d b}$ vs. genetic background-matched misty $\left(\right.$ Dock $\left.^{7 m}\right)$ mice, although to a lesser degree than in DIO. To determine whether similar patterns are observed in human subjects, we analyzed RNA-Seq data from liver biopsies from a cohort of lean, obese (without T2D), and obese, T2D patients $(n=4 \text { per group })^{29}$. Consistent with our mouse studies, obesity and T2D in humans were associated with repressions in lncRNAs vs. mRNAs (Fig. 1e, f and Supplementary Data 5).

To determine if our metabolically regulated lncRNAs represent liver-enriched transcripts, we performed RNA-Seq in seven tissues of lean $\mathrm{C} 57 \mathrm{BL} / 6 \mathrm{~N}$ mice and identified clusters of lncRNAs enriched in each tissue (Supplementary Fig. 1f). Within the liver-enriched cluster, seven DIO-associated lncRNAs (termed L-DIO-lncRNAs), including 4833411C07Rik, 9030616G12Rik, 9030622O22Rik, B930025P03Rik, Gm10319, Gm8883, and previously reported $\operatorname{lncLSTR}{ }^{24}$ (Supplementary Fig. 1g) matched our criteria: the expression of L-DIO-lncRNA expression was confined to hepatocytes compared with 
a
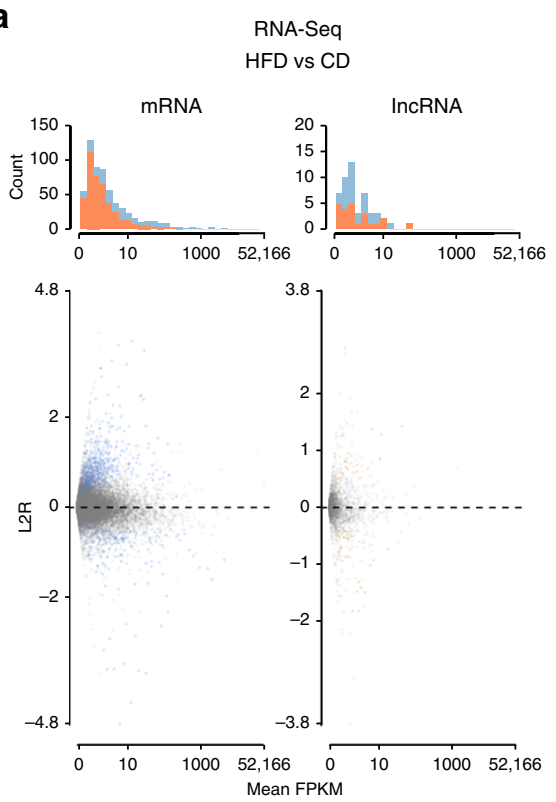

C

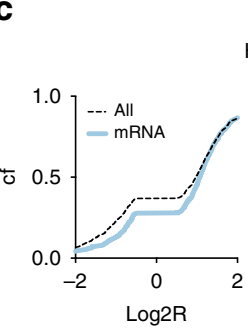

RNA-Seq

HFD vs CD

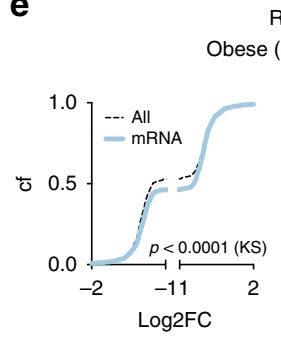

RNA-Seq

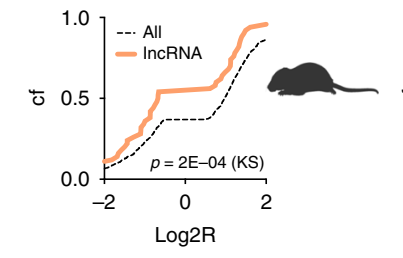

Obese ( $\odot T S D)$ vs Lean
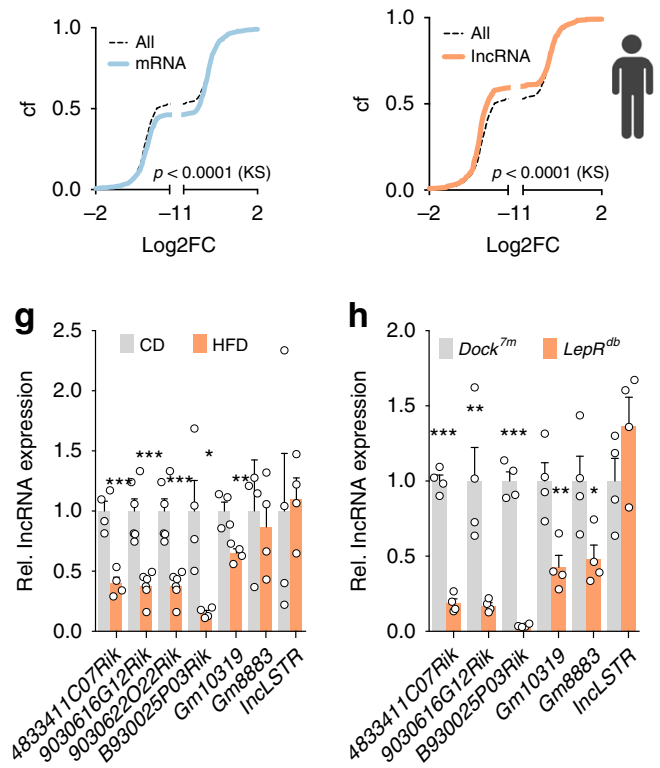

non-parenchymal liver cell types (Supplementary Fig. 1h). With the exception of Gm8883, L-DIO-lncRNAs were located in the nucleus (Supplementary Fig. 1i), and with exception of Gm10319, exhibited low protein-coding potential (using two independent algorithms, CPAT ${ }^{30}$ and $\mathrm{CPC}^{14}$, Supplementary Fig. $1 \mathrm{j}, \mathrm{k}$ ). Using quantitative polymerase chain reaction (qPCR) we confirmed L-DIO-lncRNAs to be reduced by HFD vs. CD b

RNA-Seq

AL vs FA
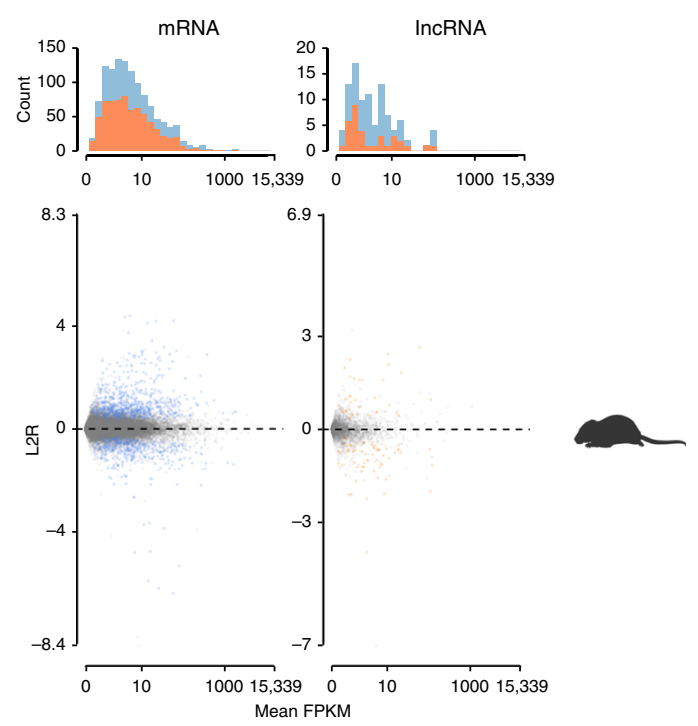

d

RNA-Seq

AL vs FA
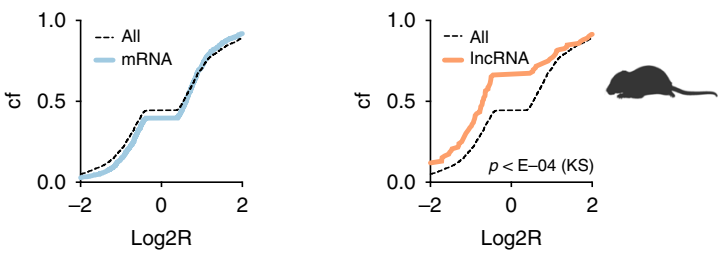

$\mathbf{f}$

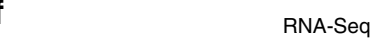

Obese $(+\mathrm{T} 2 \mathrm{D})$ vs Lean
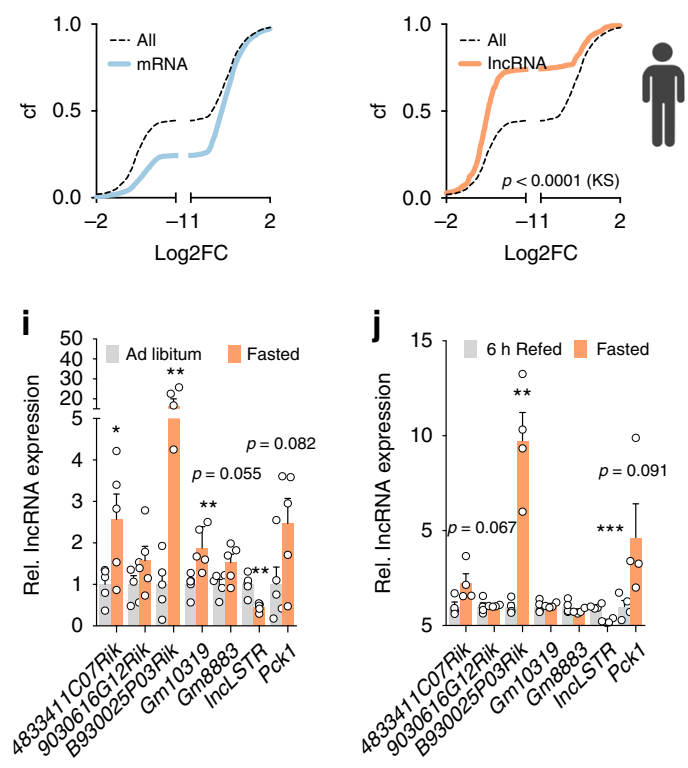

feeding (Fig. 1g), in $L e p R^{d b}$ vs. Dock ${ }^{7 m}$ (Fig. 1h), in AL vs. FA (Fig. 1i), and in RF vs. FA mice (Fig. 1j). Thus, our extensive RNA-Seq analyses and qPCR validation identified an inverse correlation of nutrient levels with lncRNAs across several mouse models of altered energy homeostasis and metabolically compromised humans and identified metabolically regulated (liver-selective) lncRNAs. 
Fig. 1 Systemic nutrient states elicit opposing effects on liver mRNA and IncRNA expression. a, b Histogram plot of read counts (top), scatter plot of reads counts vs. log2-transformed expression ratios (Log2R, bottom) and c, d cumulative frequency distribution (Cf) of Log2R of hepatic protein-coding mRNA (blue) and IncRNA (orange) expression changes. Data are from total RNA-Sequencing (RNA-Seq) in the liver of C57BL/6N mice after a, $\mathbf{c} H F D$ vs. (vs) CD feeding ( $n=3$ each) or $\mathbf{b}$, $\mathbf{d}$ ad libitum feeding (AL) vs. $16 \mathrm{~h}$ of fasting (FA) ( $n=4$ each). e Log2R Cf of mRNA (blue) and IncRNA (orange) expression changes in the liver from lean (L) vs. obese (OB) patients without T2D ( $n=4$ per group). $\mathbf{f} \log 2 \mathrm{R}$ Cf of mRNA (blue) and IncRNA (orange) expression changes in the liver of lean (L) vs. obese patients with T2D ( $n=4$ per group). g-j Quantitative reverse-transcription (RT) polymerase chain reaction (qPCR) for L-DIO-IncRNA expression in mice exposed to $\mathbf{g}$ HFD vs. CD feeding $(n=4-6)$. h Leprdb vs. Dock $7 m(n=4)$. i AL vs. FA $(n=5$ each) and j RF vs. FA ( $n=4$ each). c-f Statistical differences between mRNAs and IncRNAs were assessed using nonparametric Kolgomorov-Smirnov (KS) tests. $p$ Values are given in the panels. Bar graphs represent mean \pm s.e.m. with all data points plotted and differences in (g-j) were calculated using unpaired, twotailed Student's $t$ tests. ${ }^{\star} p<0.05,{ }^{\star \star} p<0.01,{ }^{\star \star \star} p<0.001$. Source data are provided as a Source Data file. Icons in a-f were created with BioRender.com.

Liver MAFG links high nutrient states to lncRNA repression. Our data suggested that many lncRNAs are discordantly affected by (patho)-physiological changes in nutrient states when compared with mRNAs. We hypothesized that these differences between mRNAs and lncRNAs could reflect differences in TFbinding site (TFBS) occurence in promoters of both gene sets. These differences in promoter architecture could, in turn, be differentially transactivated by nutrient-sensitive signaling pathways, leading to anticorrelative regulation of lncRNAs vs. mRNAs during obesity and T2D as observed. Our hypothesis built on in silico analyses of chromatin-state maps ${ }^{31}$ and validation studies in human cell lines ${ }^{32}$ that suggest preexisting promoter differences between lncRNAs and mRNAs. To identify TF pathways that control lncRNAs and mRNAs via distinct regulatory programs, we first analyzed putative promoter sequences ( $-800 \mathrm{bp}$ to $+100 \mathrm{bp}$ around transcriptional start sites, TSS) from an extended set of 1920 mRNAs and 149 lncRNAs affected by HFD ( $p$ value $<$ 0.1, CuffDiff DGE output). Next, we used $\mathrm{AME}^{33}$ (MEME suite $^{34}$ ) to call differences in TF motif occurence between lncRNA and mRNA promoters. Consistent with previous reports ${ }^{31,35}$, we observed that CpG-rich motifs were overrepresented in mRNA promoters, particularly motifs recognized by the E2F family of TF (e.g., E2F2-E2F4). In contrast, lncRNA promoters were enriched for MAFG:NFE2L1 ( $V$-Maf Avian Musculoaponeurotic Fibrosarcoma Oncogene Homolog G:Nuclear Factor, Erythroid 2 Like 1) motifs (Fig. 2a). This finding was consistent with reports demonstrating that TFBS recognized by MAFG or other members of the small MAF (smMAF) TF family (MAFF, MAFG, and MAFK) is overrepresented in lncRNA promoters ${ }^{31,32,35}$. In a second step and given the reported gene-repressive properties of MAFG homodimers ${ }^{36,37}$, we wanted to exclude that MAFG motifs occurred in lncRNA promoters simply because lncRNA tends to be more repressed than mRNAs during DIO. We thus performed de novo TF motif enrichment analyses of the putative promoter sequences $(-800 /+100 \mathrm{bp}$ around TSS $)$ in HOMER $\mathrm{H}^{38}$ separately for induced and repressed genes, and confirmed that smMAF motifs were enriched in lncRNA promoters affected by DIO vs. protein-coding RNAs in both gene sets (Supplementary Fig. 2a, b). Taken together, our data suggested that MAFG, or other smMAF TFs, is enriched in lncRNA promoters as compared with mRNAs, and could elicit anticorrelative transcriptional effects, for instance repress hepatic lncRNAs while, at the same time, inducing levels of specific mRNAs.

Because smMAF TF is considered as functionally redundant ${ }^{39}$, we next asked which smMAF constituted the prevalent liver isoform, and presumably, exerted the strongest effect on lncRNA repression during obesity. Using RNA-Seq, we found that $\mathrm{Mafg}$ accounted for the majority of smMAF transcripts in livers from lean C57BL/6N and Dock ${ }^{7 m}$ mice (Fig. 2b). Integrating RNA-Seq analysis from mice injected with Mafg cDNA-expressing adenoviruses (Ad-MAFG), and public MAFG chromatin immunoprecipitation coupled to sequencing (ChIP-Seq) datasets ${ }^{40}$, we found that MAFG preferentially bound lncRNAs repressed during DIO (Fig. 2c). We performed qPCR in livers from AdMAFG-treated mice and confirmed that the seven L-DIOlncRNAs (Fig. 2d) were repressed after Mafg overexpression. Conversely, locked nucleic acid (LNA)-mediated knockdown of Mafg (Supplementary Fig. 2c), but not Maff or Mafk (Supplementary Fig. 2c-e), in primary hepatocytes derepressed a set of lncRNAs containing MAFG-binding sites (Fig. 2e) as determined by ChIP-Seq (Fig. 2f). These data supported the hypothesis that MAFG represses specific lncRNAs in DIO, suggesting MAFG as TF whose activity changes during metabolic states. To investigate the degree of smMAF signaling activity in lean and obese livers, we applied ISMARA ${ }^{41}$, a web-based tool modeling genome-wide expression changes in RNA-Seq data by predicting the underlying combination of TFBS. As proof of principle and as expected, we found an induction of inflammatory CEBPB and RELA-RELNFKB1 gene programs in obese liver (Supplementary Fig. 2f, g). When comparing all combinations of nutrient-rich (HFD, AL, and RF) vs. nutrient-poor conditions (CD and FA) we found that anabolic conditions exhibited higher smMAF (NFE2:BACH1: MAFK, MAFG:NFE2L1, and MAFF) motif activities in the liver, while large $\mathrm{MAFA}^{42}$-dependent TFBS activities were reduced after DIO or RF (Fig. 2g). Importantly, RNA-Seq from HFD and Ad-MAFG-treated mice revealed a significant overlap of directional gene expression changes and overlapping gene categories (Fig. 2h, left), while Ad-MAFG and fasting gene sets showed anticorrelative trends (Fig. 2h, right). Finally, RNA-Seq in AdMAFG livers revealed trends toward repression of lncRNAs compared with mRNAs (Fig. 2i, Supplementary Data 6). Thus, our data suggested that acute (RF and $\mathrm{AL}$ ) and chronic (DIO) nutrient exposure is associated with increased smMAF signaling, and that gain of function of the most abundant smMAF isoform in the liver, MAFG, resulted in preferential repression of lncRNAs in the liver.

Hepatic MAFG links energy states to glucose metabolism. SmMAFs recruit Cap'n'Collar protein like NFE2L1/2 (or NRF1/2) to antioxidant response elements of xenobiotic enzymes ${ }^{43}$ to govern bile acid homeostasis ${ }^{40}$ and hepatic lipid and amino acid metabolism ${ }^{44}$. To determine if MAFG is required to mediate metabolic changes during DIO, we first used an in vitro approach: using LNA transfection, we silenced $M a f g$ in primary hepatocytes and observed robust suppression of Mafg mRNA and protein (Fig. 3a). To mimic fasting or RF in vitro, we treated cells with Forskolin plus Dexamethasone (FD) or FD combined with insulin (Ins, termed FDI), as reported ${ }^{45}$. At baseline, silencing $\mathrm{Mafg}$ increased glucose production (Fig. 3b). Treating cells with FD increased glucose production as expected, yet increases in glucose production remained higher when Mafg was silenced, suggesting increased gluconeogenesis. Importantly, no in vitro differences in hepatic insulin sensitivity were observed, as insulin treatment reduced glucose production in control ( $\mathrm{Scr}$ ) and Mafg LNA- treated cells to similar extents (Fig. 3b). In line with increased glucose production, Mafg RNAi increased basal 
a
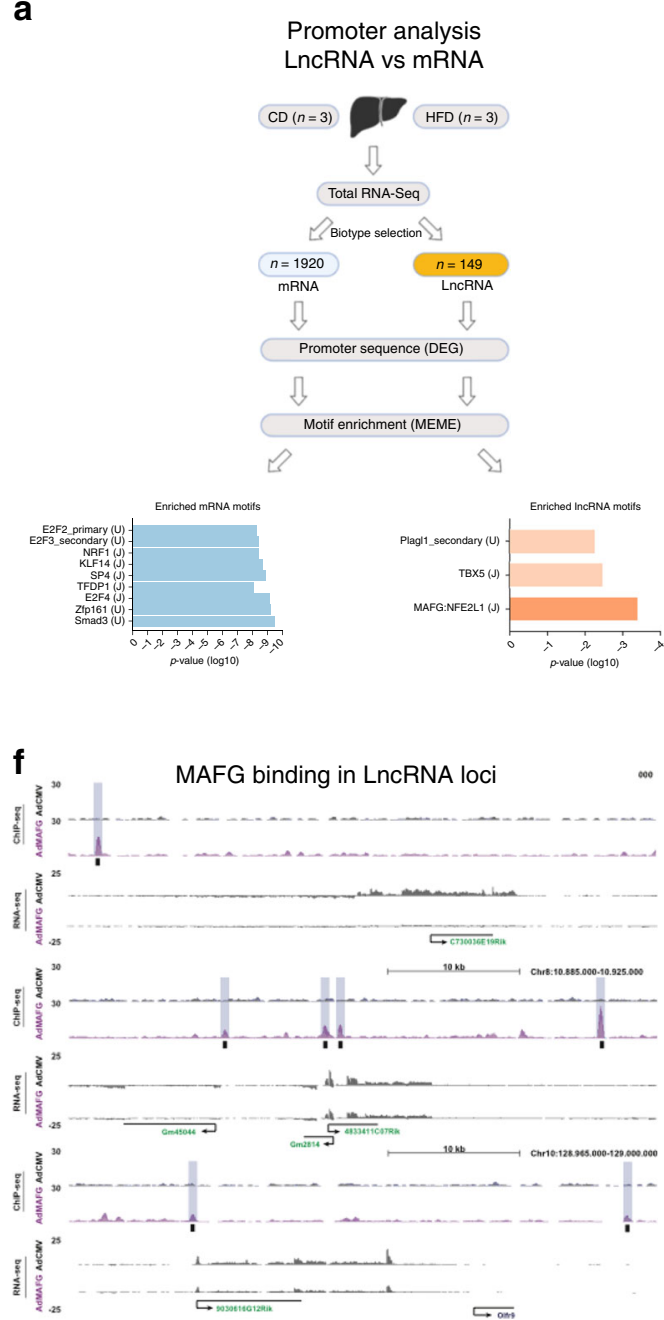

g

TF motif activity (ISMARA)
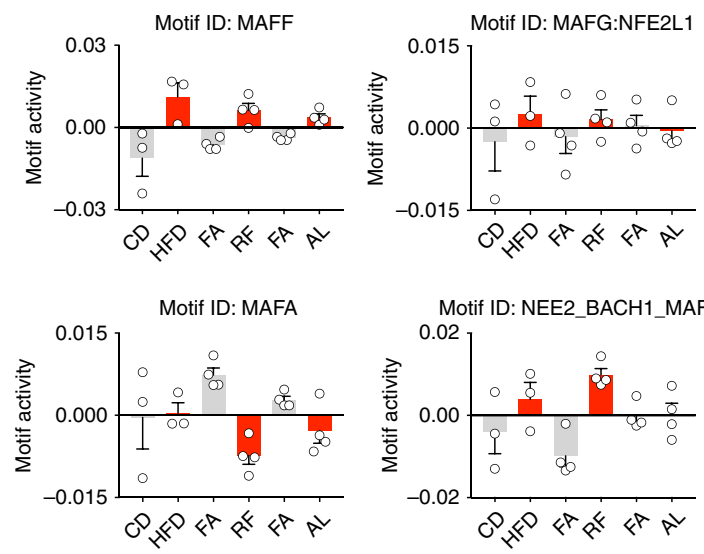

b

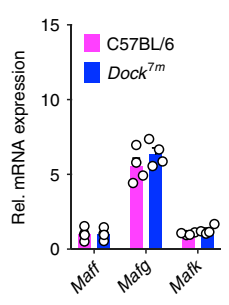

C

De Aguiar Vallim et al (GSE77559) $\begin{array}{ll}\text { Ad-MAFG } & \text { Ad-MAFG } \\ \text { (ChiP-Seq) } & \text { (RNA-Seq) }\end{array}$

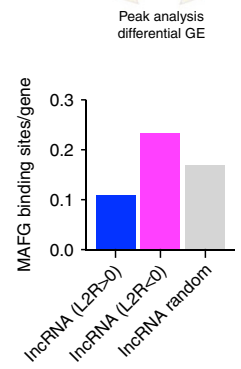

d
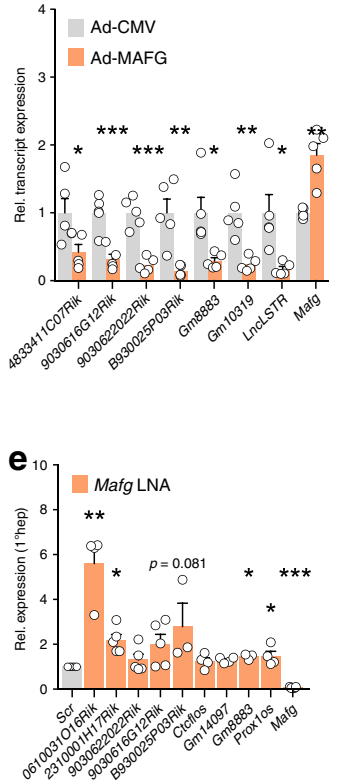

h

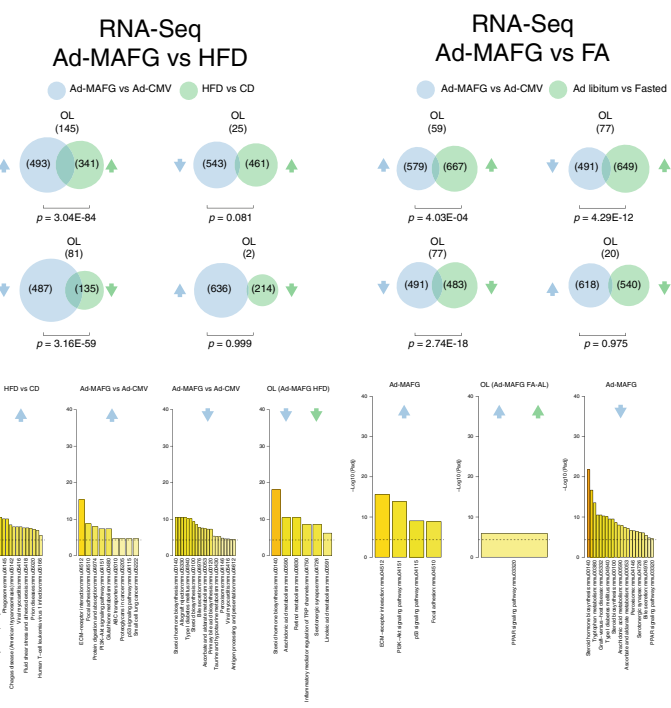

i

RNA-Seq Ad-MAFG vs Ad-CMV
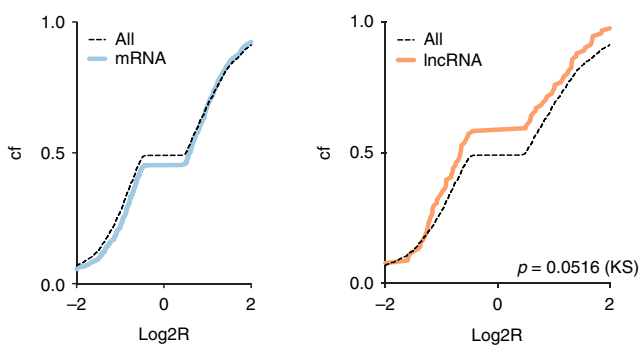

relative glucose production during a PTT, providing in vivo evidence of elevated gluconeogenesis (Fig. 3d). Importantly, LNA-mediated silencing of other smMAFs (Maff and Mafk) did not increase glucogenic gene expression (Supplementary Fig. $2 \mathrm{~h}-\mathrm{j}$ ), emphasizing specific roles for MAFG in controlling hepatic glucose output. In line with unaltered suppression of glucose production (Fig. 3b), insulin-mediated suppression of 
Fig. 2 Obesity-associated increase in MAFG signaling links nutrient states to IncRNA repression. a Illustration of transcription factor-binding site (TFBS) analysis and a list of TFBS enriched in promoters of mRNAs (blue) or IncRNAs (orange) affected by HFD vs. CD feeding ( $n=3$ each). TFBS is from UniProbe (U) or Jaspar (J). b qPCR of smMAF (Maff, Mafg, and Mafk) expression in C57BL/6N and Dock $7 m$ mice ( $n=4$ each). c Published BLRP ChIP-seq in livers of mice transduced with adenovirus (Ad)-overexpressing BLRP-MAFG fusion proteins (GEO ID: GSE77559) 40 was used as a proxy for MAFG cistromes in the liver. BLRP-MAFG peaks per gene were determined in a $\pm 50-\mathrm{kb}$ window around the TSS (defined as $\pm 1 \mathrm{bp}$ from gene start) of expressed, induced, or repressed IncRNAs by HFD using window function in bedtools ${ }^{75}$. $\mathbf{d}$ qPCR of L-DIO-IncRNAs and Mafg mRNA in livers of Ad-MAFG vs. Ad-CMV mice ( $n=5$ each). e qPCR of IncRNA exhibiting MAFG binding within TSS $\pm 50 \mathrm{~kb}$, as identified by BLRP-MAFG ChIP-Seq and Mafg expression after transfection of primary hepatocytes with $100 \mathrm{nM}$ Mafg or Scr LNAs, $n=4-5$ experiments, each performed in triplicates. $\mathbf{f}$ UCSC Genome Browser with expression of selected IncRNAs ("RNA-Seq") and BLRP-MAFG binding ("ChIP-Seq" from Vallim et al. ${ }^{40}$ ) in the liver. $\mathbf{g}$ Motif activity of smMAF and MAFA TFBS across nutritional states and HFD feeding analyzed using ISMARA ${ }^{41}$ ( $n=3-4$ per group). $\mathbf{h}$ Venn diagram illustrating DGE overlap and enriched gene ontology (GO) categories shared between indicated nutritional states, diets, and genotypes. $\mathbf{i}$ Log2R Cf of mRNA (blue) and IncRNA (orange) expression changes in livers of Ad-MAFG vs. Ad-CMV mice ( $n=4$ each). d, e Bar graphs represent mean \pm s.e.m. with all data points plotted, and statistical differences were calculated using $\mathbf{d}$ unpaired or $\mathbf{e}$ paired, two-tailed Student's $t$ test. $\mathbf{h}$ Significance of gene overlaps was calculated by using a hypergeometric distribution: ${ }^{\star} p<0.05$, ${ }^{\star \star} p<0.01,{ }^{\star \star \star} p<0.001$. Source data are provided as a Source Data file. Illustration in (a) was created with BioRender.com.

gluconeogenic enzymes under basal but also FD-stimulated conditions was unchanged after Mafg loss, again supporting the notion that Mafg loss of function does not affect insulin sensitivity (Fig. 3e). Consequently, when performing Western Blot analysis in insulin-stimulated hepatocytes in the presence or absence of $M a f g$, we observed modest reductions in phosphorylation of serine 473 residue of serine/threonine kinase AKT/ PKB (Fig. 3f), yet reduced phosphorylation of the serine 9 of glycogen synthase kinase 3 beta (GSK3B, Supplementary Fig. 2k), which is dispensible for $\mathrm{HGP}^{46}$. This suggested that IR-proximal kinases controlling glucose output, but not more distal events, are not affected by Mafg loss.

Another important TF pathway that translates information about nutrient states into increases in cell proliferation and protein translation is mammalian target of rapamycin $(\mathrm{mTOR})^{47}$ : we found insulin-evoked induction of serine 2448 mTOR phosphorylation (Fig. 3g) and concomitant increases in mTOR Complex 1 (mTORC1) activity, as evidenced by mTORC1dependent threonine 37/46 phosphorylation of eIF4E-binding protein 1 (p4E-BP1, Fig. $3 \mathrm{~h})^{48}$, blunted after Mafg silencing, suggesting that MAFG supports mTORC1-dependent processes like ribosome biogenesis and cap-dependent translation ${ }^{49}$ after insulin stimulation. Thus, we showed that increases in MAFG signaling (e.g., in DIO and after RF) preferentially repressed lncRNAs in primary hepatocytes, while LNA-mediated loss of Mafg induced lncRNAs, elicited a fasting-like expression profile, and caused specific defects in insulin-dependent activation of signaling pathways linked to protein translation.

MAFG loss protects from obesity-induced hyperglycemia. Having identified MAFG as an important regulator of lncRNA expression, and showing that Mafg repressed glucose output in hepatocytes, we next turned to in vivo loss of function. Given the obesity-evoked increase in MAFG signaling, we hypothesized that Mafg loss in vivo could be metabolically favorable in DIO and conducted Mafg knockdown by performing biweekly injections of Mafg or Scr LNAs in CD- or HFD-fed C57BL/6N mice. Mafg treatment was well tolerated over the duration of 12 weeks and caused suppression of Mafg mRNA and protein expression in lean and obese liver (Fig. 4a, f) and kidney (Supplementary Fig. 3a), but did not affect liver Maff or Mafk expression in the liver (Supplementary Fig. 3b). When studying the metabolic consequences of Mafg silencing, we did not observe alterations in body weight (BW) in CD (Fig. 4b) or HFD-fed (Fig. 4g) mice. Mafg knockdown modestly reduced fasting blood glucose $(p=0.073)$, but did not have an effect on blood glucose concentrations in the fed state (Fig. 4c), improved glucose tolerance (Fig. 4d), and trended toward improved insulin sensitivity (Fig. 4e) in CD-fed mice with mor$\mathrm{e}$ pronounced effects in obese animals (Fig. 4h-j). Indirect calorimetric quantification of oxygen consumption, carbon dioxide production, respiratory exchange ratios, energy expenditure, and food intake revealed no differences between groups on either CD or HFD (Supplementary Fig. 3c-g). No histological changes between genotypes were observed concerning fat accumulation in obese livers (Supplementary Fig. 3h). Collectively, these results implicated hepatic alterations in glucose homeostasis in the observed improvement of metabolism in Mafg LNA-treated mice. When performing RNA-Seq analysis in CD-fed Scr LNA-treated vs. HFD-fed Scr LNA-treated mice, we observed 218 genes significantly altered. When we compared these gene changes to the 357 genes significantly changed by Mafg LNA vs. Scr LNA treatment in HFD-fed mice, we found $n=66$ genes shared between both groups (Fig. 4k and Supplementary Data 8 and 12). Interestingly, we found that genes were induced by DIO as often reduced by Mafg LNA treatment. Particularly the DIO-associated increase in inflammatory gene categories was repressed in Mafgdeficient obese livers (Fig. 4k). These transcriptome analyses suggested that Mafg inhibition reinstated a healthy, less inflammatory expression profile in livers of obese mice, exemplified by trends toward increased serum fibroblast growth factor 21 (FGF21) levels (Fig. 4l), a hepatokine beneficial in the context of obesity in mice ${ }^{50}$. Furthermore, we observed trends $(p=0.103)$ toward global lncRNA derepression (Fig. 4m) as expected from Mafg RNAi in vitro.

To corroborate our findings of improved metabolic control upon Mafg silencing during obesity using independent approaches, we repeated our RNAi studies using N-acetyl galactosamine (GalNAc)conjugated Mafg and Control antisense oligonucleotide (ASO) inhibitors. In addition, given the presence of three, presumably functionally redundant ${ }^{51}$, smMAF isoforms in the liver, we silenced Mafg in the liver of HFD-fed C57BL/6 or global $\mathrm{MafF}^{-/-} / \mathrm{MafK}^{-/-}$ double-knockout (DKO) mice. After 2 weeks of initial ASO administration, mice were fed HFD for 18 weeks. As for LNAevoked Mafg ablation, Mafg ASO sustainably reduced Mafg mRNA and protein not only in the liver, but also in the kidney, vWAT, and SKM (Fig. 4n), yet did not affect Maff and Mafk expression (Supplementary Fig. 3i). Also similar to our previous study by using Mafg LNAs, in the absence of overt BW changes (Fig. 4o), Mafg loss improved insulin sensitivity (Fig. 4p, q), yet no changes in glycemia, insulin, and triglyceride levels were observed (Supplementary Fig. 3j). Crucially, ablating Mafg upon preexisting Maff/Mafk deficiency resulted in further improvements in insulin sensitivity (Fig. 4r). Thus, we here demonstrate that Mafg loss using two independent RNAi approaches improved glucose metabolism and insulin sensitivity, particularly in obese mice.

LincIRS2 knockdown causes insulin resistance in lean mice. Having demonstrated that loss of MAFG not only improved 
a

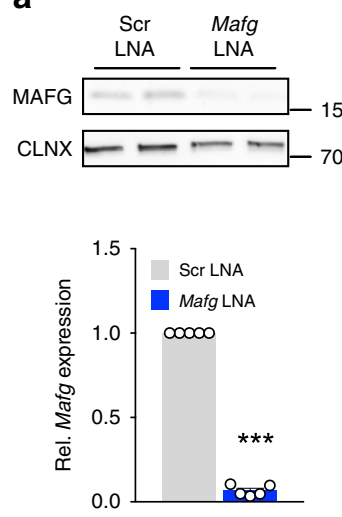

b

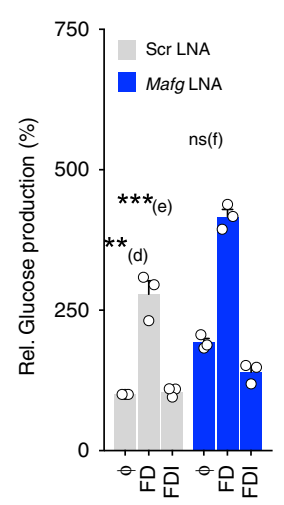

C

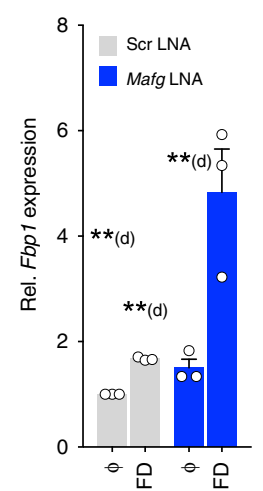

e

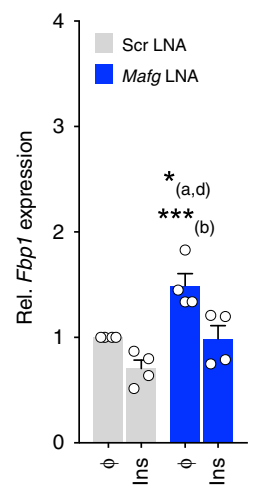

Gluconeogenesis
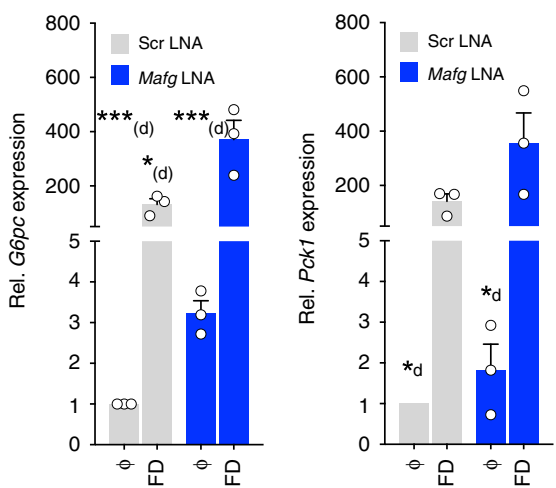

d

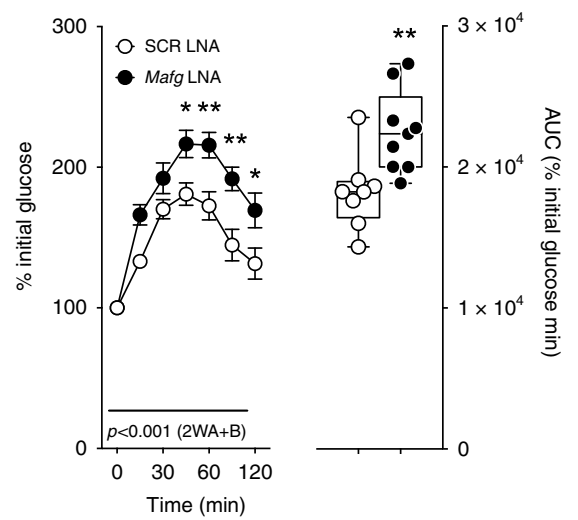

$\mathbf{f}$

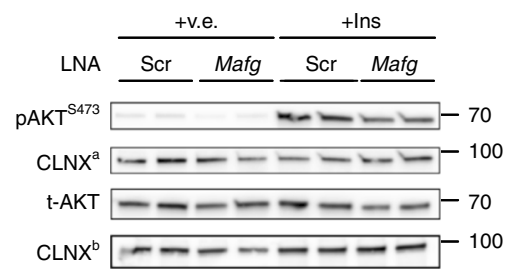

g

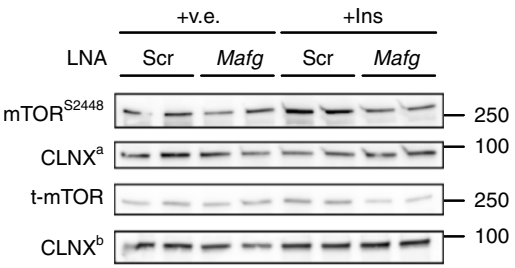

h

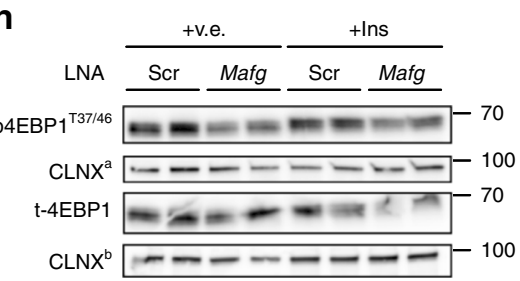

\section{Gluconeogenesis}
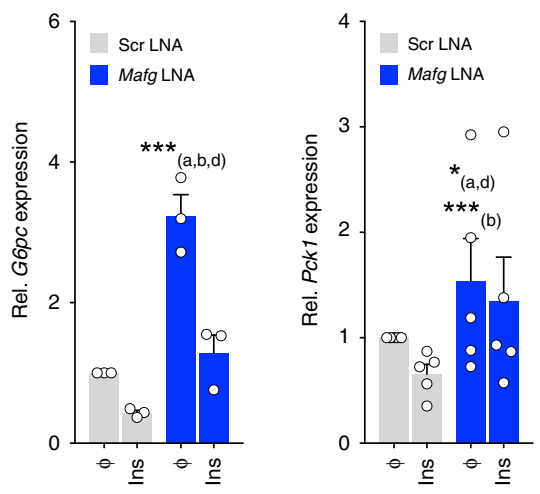

glucose homeostasis and insulin sensitivity but also, concurrently, controlled lncRNA abundance in the liver, we asked if MAFGrepressed lncRNAs are implicated in liver metabolism focused on characterizing MAFG-dependent L-DIO-lncRNA 4833411C07Rik, which we refer to as "LincIRS2" due to its positioning $80 \mathrm{~kb} \mathrm{5'} \mathrm{of}$ Irs2 (Supplementary Fig. 4a) in the following. As expected from our previous Mafg overexpression data, LincIRS2 was induced in livers from Mafg LNA and Mafg ASO-treated C57BL/6N mice (Fig. 5a). As obesity represents a complex scenario of dysregulated glucose, insulin, lipid, and hormone homeostasis that all affect liver energy metabolism ${ }^{1}$, we investigated which obesity-related nutrients, hormonal factors, and nutrient-sensitive TFs, in addition to MAFG, regulated LincIRS2. For this, we expressed constitutively active (ca) versions of metaboregulatory TFs in primary hepatocytes, including glucose-sensing MLX-interacting protein like/carbohydrate response element-binding protein (caMLXIPL/ $\mathrm{ChREBP}^{52}$ ), FOXO1 (caFOXO11 ${ }^{53}$ ), or lipogenic caSREBP1C ${ }^{54}$. We found that LincIRS2 was induced upon catabolic caFOXO1 and caChREBP expression in primary hepatocytes (Fig. 5b) in addition to fasting-mimicking cyclic adenosin-3',5'-monophosphate (cAMP) and glucagon (GCG) stimulation (Fig. 5c). On the other hand, insulin (with or without glucose co-stimulation) repressed LincIRS2 (Fig. 5d), whereas IR knockout in liver induced LincIRS2 (Fig. 5e). Concomitantly, constitutive active versions of (insulin-dependent) SREBP1C (caSREBP1) decreased LincIRS2 expression (Fig. 5f). Thus, LincIRS2 inversely correlates with MAFG and insulin signal transduction across several in vitro and in vivo models of altered signaling and nutritional states. 
Fig. 3 MAFG signaling in hepatocytes represses glucose production and supports mTOR activation. a Representative MAFG immunoblot and Mafg expression after transfection of primary hepatocytes with $100 \mathrm{nM}$ Mafg or Scr LNA. b Relative glucose levels in primary hepatocytes after transfection with $100 \mathrm{nM}$ Mafg or Scr LNAs and stimulation for $24 \mathrm{~h}$ with $10 \mu \mathrm{m}$ Fsk plus $100 \mathrm{nM}$ Dex (FD) or FD combined with $100 \mathrm{nM}$ insulin (FDI). c, e Indicated mRNAs after transfection with Mafg or Scr LNAs under basal conditions or stimulated with c $10 \mu \mathrm{M}$ Fsk plus $100 \mathrm{nM}$ Dex (FD) or e $100 \mathrm{nM}$ insulin. Data represent $\mathbf{a} n=5$ or $\mathbf{b}, \mathbf{c}$, e $n=3-4$ independent experiments, each performed in triplicates. d Pyruvate tolerance test (PTT) performed in C57BL/6N mice after 6 weeks of HFD feeding with $10 \mathrm{mg} \mathrm{kg}^{-1}$ of Mafg $(n=9)$ or $\operatorname{Scr}(n=8)$ LNA injected 5 days before. $\mathbf{f}-\mathbf{h}$ Immunoblots of $\mathbf{f}$ total and Serine 473 phosphorylated AKT/PKB (pAKT ${ }^{\mathrm{S} 473}$ ). $\mathbf{g}$ Total and Serine 2448 phosphorylated mammalian target of rapamycin (pmTOR ${ }^{\mathrm{S} 2448}$ ) and $\mathbf{h}$ total and Threonine $37 / 46$ phosphorylated eukaryotic translation initiation factor $4 \mathrm{E}$ binding protein 1 ( $\mathrm{p} 4 \mathrm{~EB}-\mathrm{BP} \mathrm{1}^{37 / 46}$ ) after transfection of primary hepatocytes with $100 \mathrm{nM}$ Mafg or Scr LNAs and stimulated for 10 min with vehicle (v.e.) or $100 \mathrm{nM}$ insulin. Separate membranes were loaded with equal amounts of protein lysate for total and phospho-specific immunoblotting, and equal loading was confirmed by calnexin (CLNX) for each membrane. Due to different molecular weights of $\mathrm{mTOR}, \mathrm{PKB} / \mathrm{AKT}$, and $4 \mathrm{E}-\mathrm{BP1}$, these proteins were partly investigated on the same membranes together with the same CLNX antibody. CLNXa and $C L N X^{b}$ bands are therefore identical. Each blot is representative of $n=3$ immunoblots, each immunoblot performed after transfection of Mafg vs. Scr LNA in hepatocyte preparations from $n=2$ mice. a-c, e Bar graphs represent mean \pm s.e.m. with all data points plotted and statistical differences were calculated using a paired, two-tailed Student's $t$ test. b, c, e One-way ANOVA and d two-way ANOVA with Bonferroni post correction for multiple testing. Superscripts depict group comparisons for post analysis (a-f=comparison vs. column 1-6). d Box plot bounds depict upper and lower quartiles with median as the center. Whiskers span all values: ${ }^{\star} p<0.05,{ }^{\star \star} p<0.01,{ }^{\star \star \star} p<0.001$. Source data are provided as a Source Data file.

We next asked if, like MAFG, LincIRS2 is involved in hepatic glucose control. LNA-mediated LincIRS2 knockdown in primary hepatocytes was highly efficient under basal, FD, GCG, and insulin-stimulated conditions (Fig. 5g, h). Crucially, LincIRS2deficient primary hepatocytes did not exhibit suppression of Pck1 expression upon insulin stimulation (Fig. 5i). These data suggested in vitro insulin resistance upon LincIRS2 silencing as, in contrast to Mafg, LincIRS2 RNAi did not increase Pck1 expression under basal, FD, and GCG stimulation (Fig. 5j, k).

We next investigated the consequences of LincIRS2 knockdown in vivo by performing biweekly injections of LNAs targeting LincIRS2. LincIRS2 silencing reduced 50\% of liver and kidney LincIRS2 in lean mice (Fig. 5l), and again, we observed no differences in BW between genotypes in CD- or HFD-fed mice (Fig. 5m, n). LincIRS2 LNA-treated lean mice were normoglycemic (Fig. 5o, left), yet mildly insulin resistant (Fig. 5o, right), whereas LincIRS2 LNA-treated obese mice were unaffected, presumably due to preestablished insulin resistance in these animals (Fig. 5p). In line with this, LincIRS2 RNAi reduced insulin-mediated AKT/PKB phosphorylation of serine 473 in livers from lean (Fig. 5q) but not obese (Fig. 5r) mice in the absence of changes to glucose tolerance (Supplementary Fig. $4 \mathrm{~b}$ ) or hepatic lipid accumulation (Supplementary Fig. 4c). RNA-Seq analysis of LincIRS2 LNA-treated livers confirmed the deterioration of metabolism at the transcriptome-wide level as DGE in lincRS2-deficient lean mice revealed similar gene sets affected as in Scr LNA-treated obese mice (Supplementary Fig. 4d, Supplementary Data 7 and 9).

Knockout of LincIRS2 causes elevated glucose levels in mice. Given the mild deterioration of metabolism upon transient LincIRS2 knockdown in LNA-treated mice, we wanted to address the role of LincIRS2 in glucose metabolism using a genetic model. For this, we employed CRISPR/Cas9 genome editing in order to target a 418-bp region encompassing exon 1 and the putative promoter region (marked by H3K4me3 and H3K9Ac, Supplementary Fig. 4f-i) of LincIRS2. Sanger sequencing of the LincIRS2 allele confirmed a 426-bp deletion, together with a 5-bp insertion of the desired genomic LincIRS2 locus (Supplementary Fig. 4j). Homozygous C57BL/6N-LincIRS2 ${ }^{\text {em/Cecad }}$ knockout mice (termed LincIRS2 $2^{\Delta / \Delta}$ ) exhibited complete absence of LincIRS2 expression (Supplementary Fig. 4k), were born at Mendelian frequencies, and exhibited no developmental or behavioral abnormalities, changes in BW, energy expenditure, or substrate mobilization (Supplementary Fig. 4l-n). Lean LincIRS2 ${ }^{\Delta / \Delta}$ mutants exhibited elevated blood glucose levels under fasted and fed conditions (Fig. 6a) and glucose levels remained high after insulin (Fig. 6b) or glucose challenge (Fig. 6c). Interestingly, LincIRS2 ${ }^{\Delta / \Delta}$ mice showed elevated gluconeogenic G6pc, Pck1, and Foxo1 (Fig. 6d), oxidative Acyl-CoA Oxidase 1 (Acoxl) and Carnitine Palmitoyltransferase $1 A$ (Cpt1a, Fig. 6e), insulin receptor substrate (Irs) 1 and 2 (Fig. 6f), and Slc2a1-2 glucose transporter expression (Fig. 6g), suggesting altered transcriptional regulation of these enzymes underlying the elevated glycemia in these mice.

Finally, and to test if LincIRS2 synergized with MAFG function, we overexpressed Mafg using adeno-associated viruses in C57BL/6 and LincIRS2 $\Delta / \Delta$ mice. Adeno-associated virus serotype 8 (AAV8)-mediated overexpression (AAV8-MAFG) increased Mafg mRNA and protein (Fig. 6h), but did not alter fasted or random-fed glucose levels (Fig. 6i, j). Intriguingly though, driving Mafg, alone or coupled to LincIRS2 loss, again caused glucose intolerance to similar extents, arguing for synergistic roles of MAFG overexpression and LincIRS2 deletion (Fig. 6k, l) in glucose metabolism. Conversely, delivery of $\mathrm{Mafg}$ alone caused insulin resistance (Fig. $6 \mathrm{~m}$ ), but not when coupled to LincIRS2 loss (Fig. 6n), suggesting independent roles in regulating insulin sensitivity. Taken together, our data identified a transcriptional pathway where MAFG acts as a repressor of metabolically regulated hepatic lncRNAs. We identified a specific MAFG-repressed lncRNA, LincIRS2, that contributed to glucose homeostasis and insulin-mediated suppression of hepatic glucose production in mice. Either genetic LincIRS2 deletion or LincIRS2 RNAi in vivo impaired glucose metabolism, albeit to different degrees, and presumably, via different molecular mechanisms.

Collectively, we demonstrated a hitherto unknown role for MAFG in serving as intracellular sensor of systemic nutrient states, where changes in MAFG signal transduction are coupled to alteration of hepatic glucose homeostasis. Further, increased MAFG signaling partly explained the initial observation of opposite regulation of lncRNAs vs. mRNAs during DIO and T2D. One molecular effector mechanism of how excessive MAFG activity during DIO translated into glucose alterations is repression of liver-selective LincIRS2, which itself integrated anabolic and catabolic metabolites and TF responses (illustrated in Fig. 6o). In vivo genome editing and RNAi against LincIRS2 revealed roles for this MAFG-IncRNA axis in ensuring hepatic glucose handling and maintenance of insulin sensitivity.

\section{Discussion}

Our results establish the TF MAFG as a regulator of energy-rich nutrient states in the liver, both in postprandial responses and during metabolic diseases like obesity and type 2 diabetes. 
a

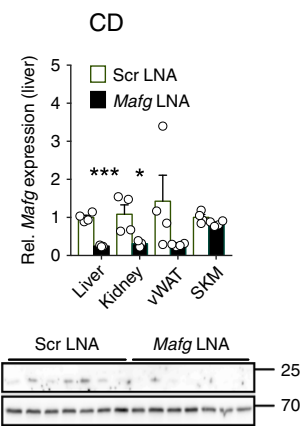

f

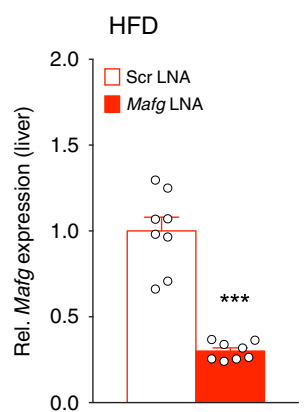

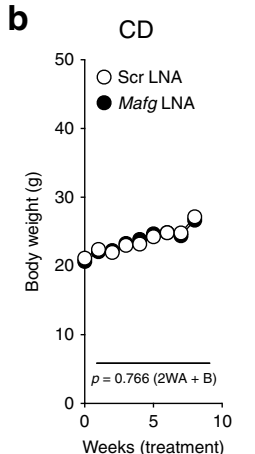

c
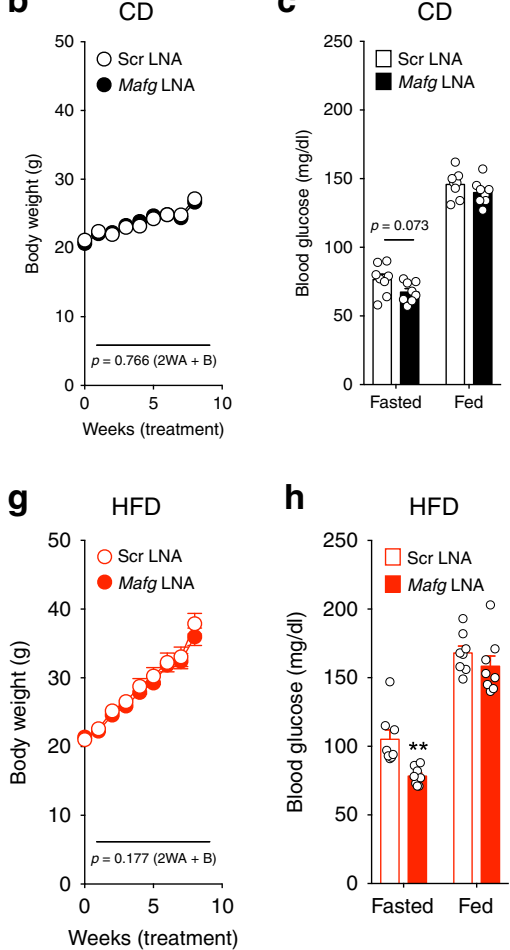

h

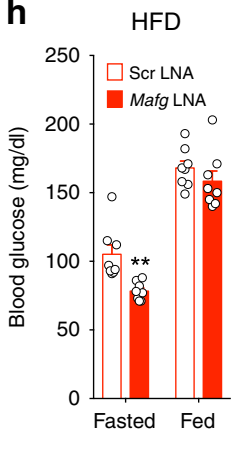

d

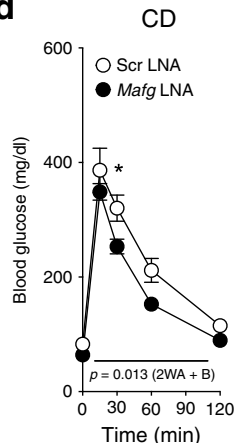

i

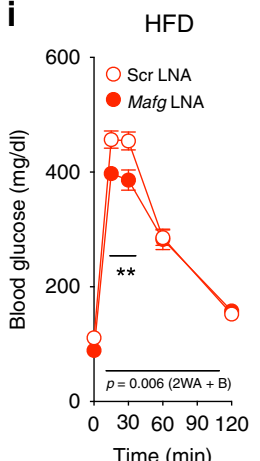

e

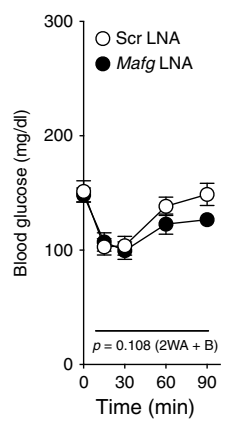

j

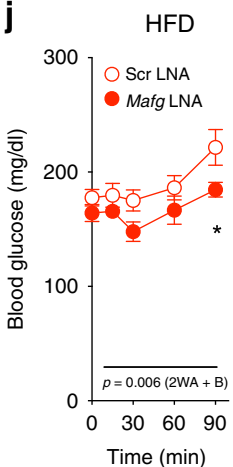

$\mathbf{k}$

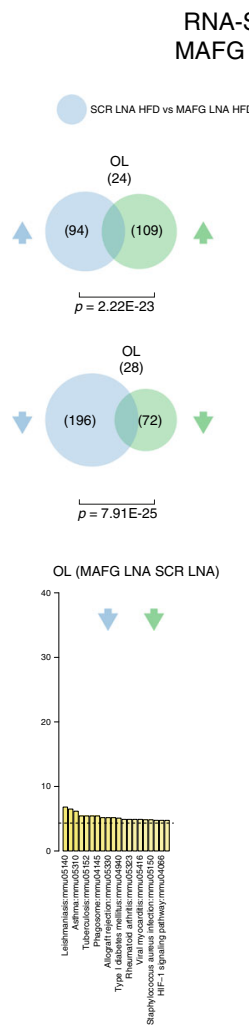

RNA-Seq Overlap AFG LNA vs HFD
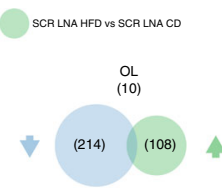

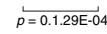

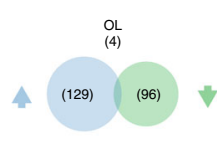

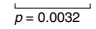
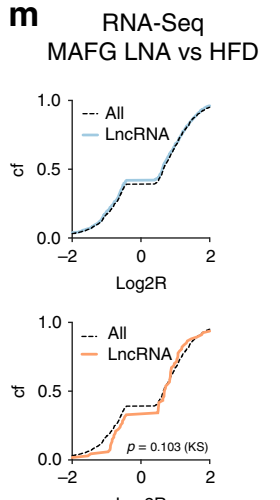

Log2R

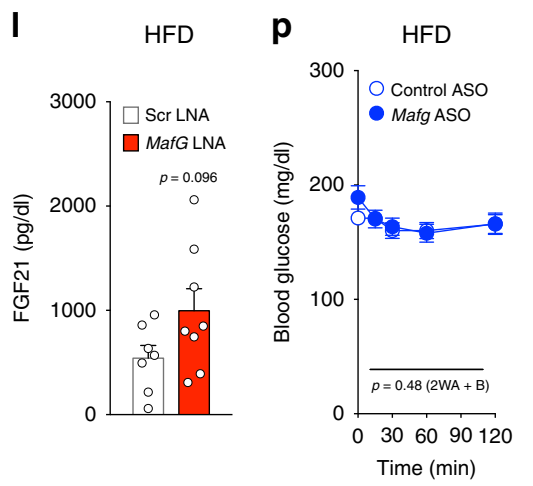

o
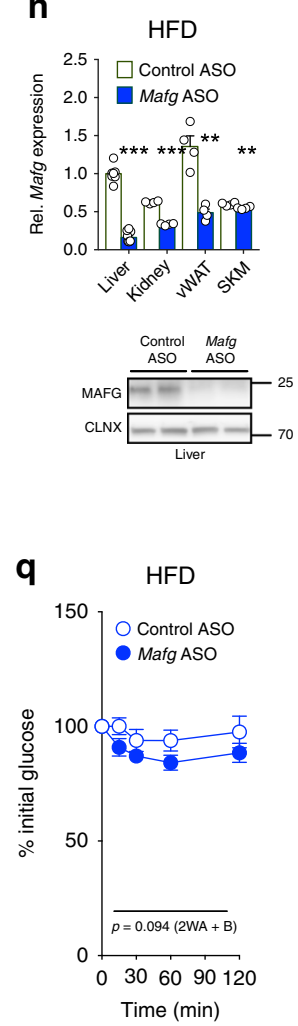
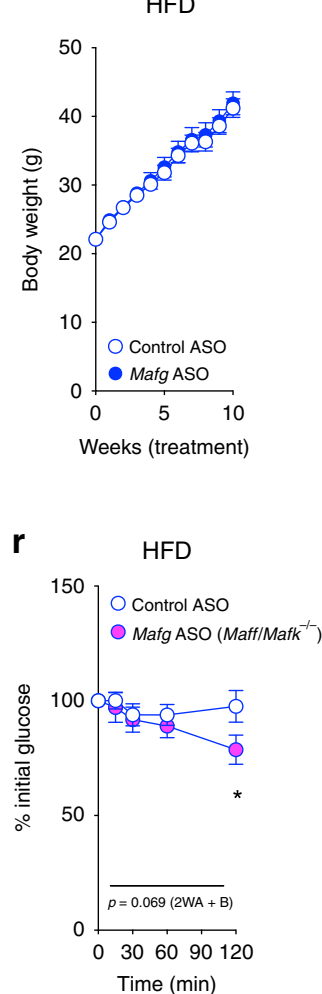

Mechanistically, we demonstrated that MAFG controls a specific set of obesity-associated hepatic lncRNAs that are repressed in obese livers, in addition to the known function of MAFG in bile acid regulation ${ }^{40}$.

Our results show little evidence for co-regulation with only four disease-associated syntenically conserved lncRNAs in human and mouse livers (Supplementary Data 13) when performing comparative lncRNA analyses using $\mathrm{PLAR}^{55}$. This may be due to
(1) misannotation of gene biotypes, as not every lncRNA transcript truly represents a noncoding transcript, evidenced, e.g., by the high protein-coding potential of IncRNA Gm10319, (2) due to the fact that lncRNA conservation at the structural and function level is hard to address computationally ${ }^{56}$, or (3) conservation of disease-associated lncRNAs simply is low as shown in other cell types ${ }^{57}$. Another caveat lies in the low number of obesityassociated lncRNAs we identified here. This limits the statistical 
Fig. 4 MAFG loss protects from obesity-induced hyperglycemia. a qPCR of Mafg expression in the liver from CD-fed 21-week-old C57BL/6N mice after 15 weeks of $10 \mathrm{mg} \mathrm{kg}^{-1}$ of Mafg $(n=8)$ or Scr LNA $(n=7)$ and immunoblot of MAFG and CLNX protein. b Body weight, c 16-h fasted, and random-fed glucose, $\mathbf{d}$ GTT, e ITT in CD-fed C57BL/6N mice after 6 and 5 weeks of Mafg or Scr LNA $(n=8)$. $\mathbf{f}$ qPCR of Mafg expression in the liver from 21-week-old HFD-fed mice after 15 weeks of Mafg or Scr LNA $(n=8)$. $\mathbf{g}$ Body weight, $\mathbf{h} 16-\mathrm{h}$ fasted, and random-fed glucose, i GTT, and $\mathbf{j}$ ITT in HFD-fed C57BL/6N mice after 6 and 5 weeks of Mafg or Scr LNA $(n=8)$. k DGE overlap and enriched categories in livers from CD- or HFD-fed C57BL/6N mice after 10 weeks of Mafg or Scr LNA $(n=4)$. I FGF21 levels from 21-week-old HFD-fed C57BL/6N mice after 15 weeks of Mafg $(n=8)$ or Scr LNA $(n=7)$. $\mathbf{m} \log 2 \mathrm{R}$ Cf of mRNA and IncRNA expression changes in 21-week-old HFD-fed mice after 15 weeks of Mafg or Scr LNA ( $n=3$ ). $\mathbf{n}$ qPCR of Mafg expression in liver and indicated tissues after 20 weeks of $5 \mathrm{mg} \mathrm{kg}^{-1}$ Mafg or Control ASO $(n=7$ Mafg and $n=10$ Control ASO in liver, $n=4$ other tissues) and immunoblot of MAFG and CLNX in the liver of HFD-fed C57BL/6N mice after 2 days of Mafg or Control ASO. $\mathbf{o}$ BW in 16-week-old HFD-fed mice after 10 weeks of Mafg or Control ASO ( $n=7$ Mafg and $n=10$ Control ASO). Absolute $\mathbf{p}$ relative $\mathbf{q}, \mathbf{r}$ glucose during ITT in HFD-fed $\mathbf{p}, \mathbf{q}$ C57BL/6N or $\mathbf{r}$ Maff/Mafk ${ }^{-1-}$ (DKO) mice after 11 weeks of Mafg or Control ASO ( $n=6$ Control ASO, $n=9$ Mafg ASO, and $n=6$ Mafg ASO in DKO). Bar graphs represent mean \pm s.e.m. with all data plotted and statistical differences calculated using $\mathbf{a}, \mathbf{c}, \mathbf{f}, \mathbf{h}, \mathbf{n}$ unpaired, two-tailed and $\mathbf{I}$ unpaired, one-tailed Student's $t$ test. $\mathbf{m}$ Kolgomorov-Smirnov $\mathbf{b}, \mathbf{d}, \mathbf{e}, \mathbf{g}, \mathbf{i}, \mathbf{j}, \mathbf{p}-\mathbf{r}$ two-way ANOVA with Bonferroni post correction. $\mathbf{k}$ Significance of gene overlaps was calculated by using a hypergeometric distribution: ${ }^{\star} p<0.05,{ }^{\star \star} p<0.01,{ }^{\star \star \star} p<0.001$. Source data are provided as a Source Data file.

power of TF motif enrichment algorithms. However, other in silico studies came to similar conclusions in terms of smMAF TFBS enrichment in long noncoding RNA promoters $31,32,35$.

In our study, we found that MAFG signaling activity was reduced during fasting, which has not been previously reported. We then analyzed both MAFG and its known interaction partner NRF1/NFE2L1 during nutrient deprivation and found that, while both Nrf1/Nfe2l1 and Mafg mRNA were mildly induced, NRF1/ NFE2L1, but not MAFG protein, was profoundly upregulated during fasting (Supplementary Fig. 5a, b). By reanalyzing publicly available hepatic RNA-Seq data performed in mice with hepatocyte-specific deletion of NRF1/NFE2L1 ${ }^{58}$ (GEO ID: GSE103949), we found that NRF1/NFE2L1 ablation resulted in strikingly similar expression profiles compared with MAFG overexpression (Supplementary Fig. 5c). These data suggested that the interaction of MAFG with NRF1/NFE2L1 constitutes an important regulatory step during fasting in the liver: recruitment of MAFG into NRF1/NFE2L1:MAFG heterodimer complexes, for instance after fasting-induced increases in NRF1/NFE2L1 protein would, in turn, reduce MAFG target gene expression (as during fasting), consistent with a notion where NRF1/NFE2L1 deficiency and MAFG overexpression increase pools of "free" MAFG homodimers with known gene-repressive functions ${ }^{36,37}$. Although we have not directly addressed the binding partners of MAFG under different nutrient stress conditions, our data suggest that competition between NRF1/NFE2L1 and MAFG is important for liver physiology: as found for MAFG gain of function $^{40}$, NRF1/NFE2L1 inactivation in the liver downregulated genes involved in cholesterol to bile acid conversion, e.g., $C y p 7 b 1^{58}$. This suggests that, whereas NRF1/NFE2L1 suppresses inflammation and promotes bile acid synthesis ${ }^{40}$, MAFG overexpression induces hepatic inflammation (Fig. 5k) and suppresses bile acid conversion ${ }^{40}$, in addition to suppressing key regulatory lncRNAs such as LincIRS2 (Supplementary Fig. 5d, e and Supplementary Data 11). Whether the MAFG-dependent regulation of lncRNAs (e.g., LincIRS2), or the MAFG-dependent regulation of coding mRNAs are key in mediating the MAFG-dependent changes in energy-rich nutrient states remains to be determined. However, our data suggest that the MAFG-dependent regulation of coding and noncoding mRNAs is complimentary, suggesting that both are required to elicit the full response.

Ultimately, our findings could also indicate that MAFG pathway inhibition, or NRF1/NFE2L1 activation, could generally favor energy-preserving processes where transcription of long noncoding RNAs does not require subsequent mRNA translation. Conversely, postprandial or obesity-associated activation of MAFG would trigger energy-demanding, anaplerotic processes like an increase in cellular proliferation and protein synthesis as a consequence of mTOR-4E-BP1 pathway activation.

\section{Methods}

Animal care and research diets. All animals were maintained on a C57BL/6N background, housed in groups of 3-5 animals per cage at $22-24^{\circ} \mathrm{C}$ on a constant 12-h light/dark cycle in a SPF-controlled facility with regular testing for pathogens. Care of animals and all mouse research was approved by and we adhered to institutional and animal-care committee ethical guidelines from local (Bezirksregierung Köln) or regional (Tierschutzkommission accession no. \$15 TSchG of the Landesamt for Natur, Umwelt und Verbraucherschutz (LANUV) NorthRhine Westphalia, Germany, internal reference no. Az-84-02.04-2016.A460) authorities. Upon weaning, mice were fed standard rodent chow (Teklad Global Rodent T.2018.R12; Harlan). For experiments involving controlled feeding paradigms, animals were allowed AL access to CD (D12450B* mod LS; Sniff) containing $62 \mathrm{~kJ} \%$ carbohydrates, $27 \mathrm{~kJ} \%$ protein, and $11 \mathrm{~kJ} \%$ fat and drinking water. DIO was achieved by feeding a HFD (D12492 (I) mod; Sniff) containing $22 \mathrm{~kJ} \%$ carbohydrates, $24 \mathrm{k} \mathrm{J} \%$ protein, and $54 \mathrm{~kJ} \%$ fat from starting at 6-7 weeks of age. Experimental mice were exposed to specific diets for 10-12 weeks and 17-18 weeks old at sacrifice unless described otherwise. Six-month-old LIRKO 59

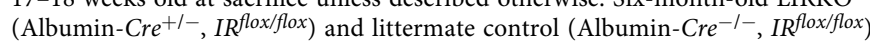
animals were used.

RNA isolation and total RNA-Sequencing. Liver samples used for total RNASequencing (RNA-Seq) were from male, 36-week-old C57BL/6N mice exposed to $\mathrm{CD}(n=3)$ or HFD $(n=3)$ feeding for 30 weeks, starting at 6 weeks of age. RNASeq of C57BL/6N liver mice exposed to different nutrient states was performed in liver samples from male, 17-week-old, lean AL $(n=4)$, AL followed by $16 \mathrm{~h}$ of fasting (FA, $n=4)$, and FA followed by $6 \mathrm{~h}$ of RF $(n=4)$ mice. For RNA-Seq from a genetic model of obesity and T2D, male BKS.Cg-Dock $k^{7 m+/+}$; LepR $R^{d b} / J(d b / d b)$ mice and BKS.Cg-Dock ${ }^{7 m+/+}$ (misty/misty) control mice were purchased from Jackson Laboratory or Janvier Labs. We used misty mice as control animals, as they are the littermates of $d b / d b$ mice generated on the BKS.Cg-Dock ${ }^{7 \mathrm{~m}+/+}$ $L e p R^{d b} / \mathrm{J}$ background ( $n=4$ mice per genotype). Tissues were collected at 10 weeks of age. RNA-Seq was also performed in lthe iver from mice treated with adenoviruses expressing Mafg cDNA (Ad-MAFG, $n=3$ ) or empty vector (Ad$\mathrm{CMV}, n=3$ ) under control of mouse cytomegalovirus (CMV) promoter ${ }^{40}$. Briefly, cDNAs for mouse MAFG, were cloned from whole-liver cDNA into pAdTrack CMV plasmids. For animal experiments, $1 \times 10 \mathrm{E} 9$ plaque-forming units were used, and tissues were collected after 5-7 days of viral treatment. RNA-Seq was also performed in the liver from 21-week-old male mice exposed to CD or HFD and injected 15 weeks with control (Scr), Mafg, or LincIRS2 LNA ( $n=4$ per genotype and diet). Human liver samples used for RNA-Seq were obtained from male individuals that were with lean and nondiabetic $(n=4$; age $60.75 \pm 9$, body mass index (BMI) $23.52 \pm 1.36$, Patient IDs: 11_0381WSC, 13_1651HRU,

135_1311WER, and 155_3141AZE), obese and nondiabetic $(n=4$; age $38.5 \pm$ 13.42, BMI $51 \pm 2.98$, Patient IDs: 31_0311RAN, 32_0861NSC, 37_1941JRO, and 98_0121STR), overweight, diabetic $(n=2$; age $75.5 \pm 0.7$, BMI $26 \pm 1.41$, Patient IDs: 54_0951LZU, 56_1781DGR, 95_1701GBO, and 152_3011FHE), and obese and diabetic $(n=2$; age $44 \pm 2.8$, BMI $48.25 \pm 20.85)$. All patients gave written informed consent, and approval of the ethics committee of the University of Ulm was obtained ${ }^{29}$. Library preparation and sequencing was performed at (1) MaxPlanck Genome-Centre (MP-GC, HFD vs. CD cohort) or (2) the Cologne Center for Genomics (CCG), Germany (others). Following quality checks, $1 \mu \mathrm{g}$ of total liver RNA of each sample was (1) depleted for rRNA using NEBNext ${ }^{\oplus}$ rRNA depletion Kit (human/mouse/rat). Library preparation was performed with NEBNext Ultra ${ }^{\text {mat }}$ Directional RNA Library Prep Kit for Illumina (New England Biolabs) or (2) depleted for cytoplasmic and mitochondrial rRNA with Ribo-Zero Gold (LNA Cohort) or Ribo-Zero and strand-specific library preparation performed using TruSeq RNA Gold Kit from Illumina. All libraries were sequenced on (1) HiSeq2500 in $2 \times 100$-bp PE or (2) HiSeq 4000 instruments in $2 \times 75 \mathrm{PE}$ sequencing mode. 

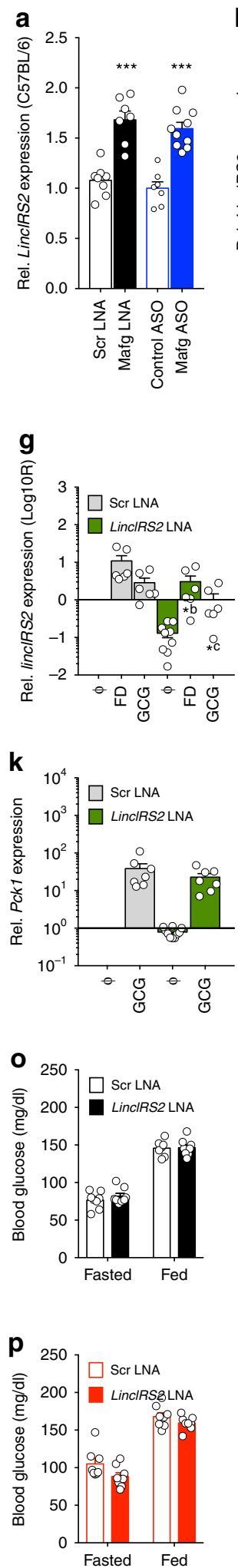
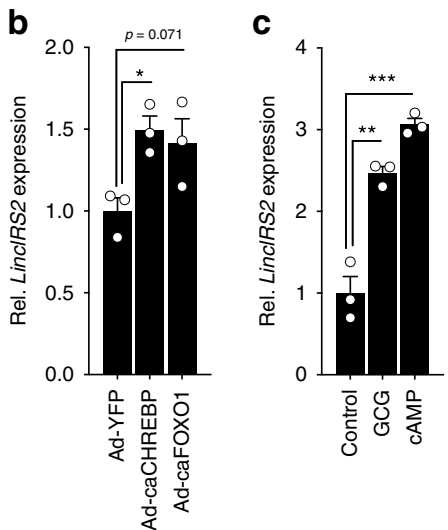
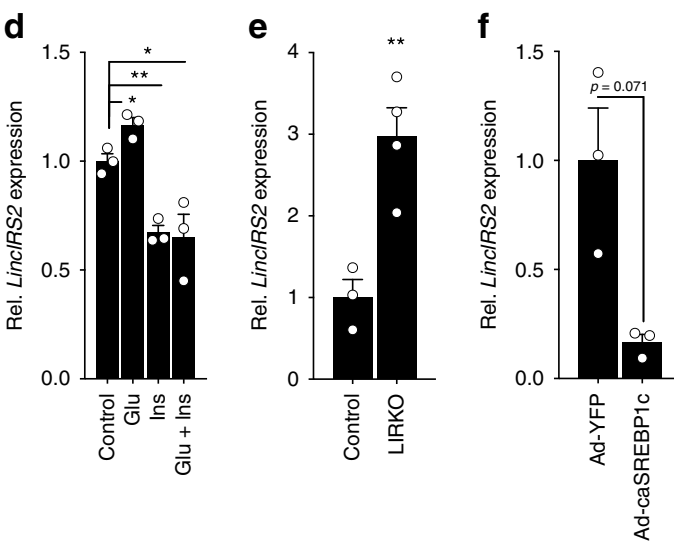

h

i

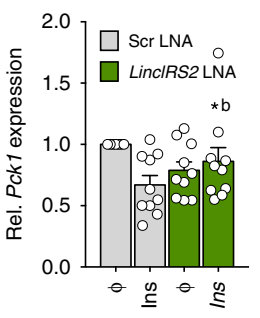

j
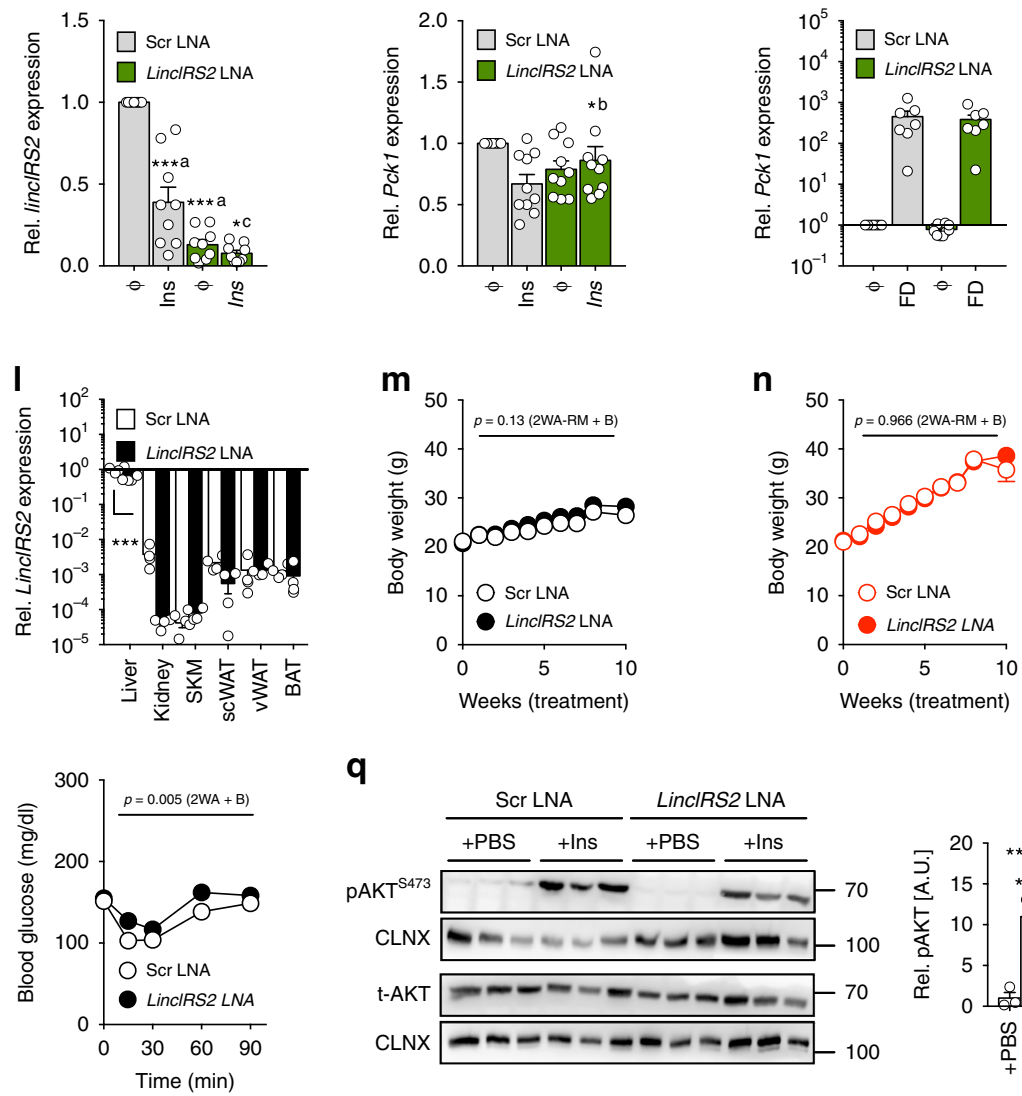

q
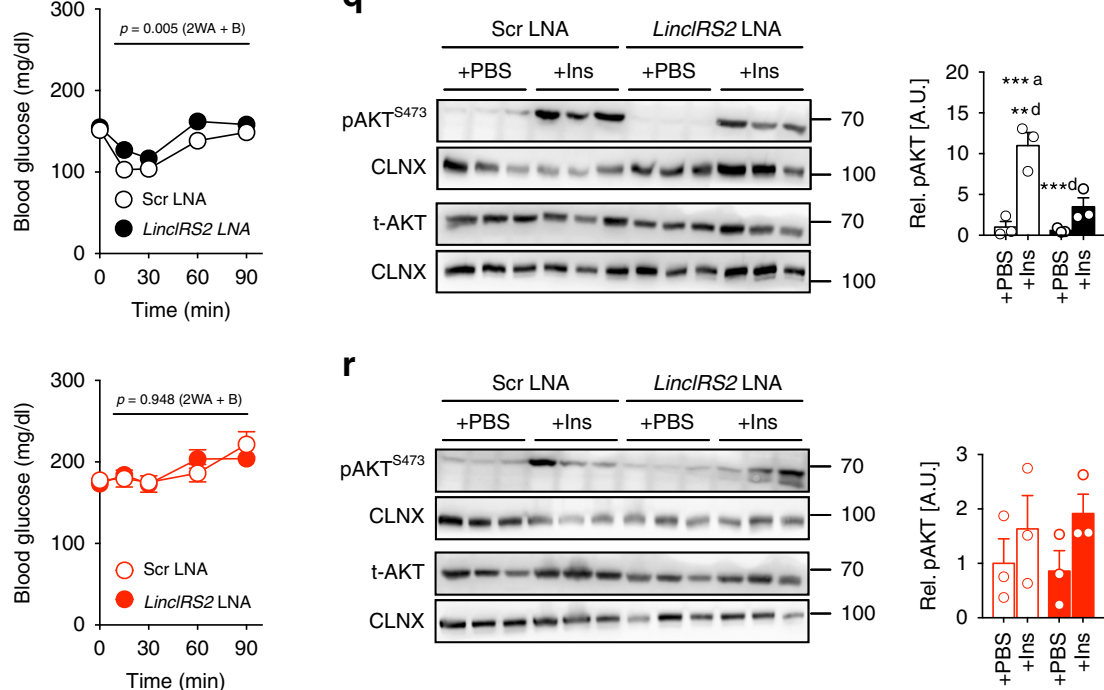

$\mathbf{r}$
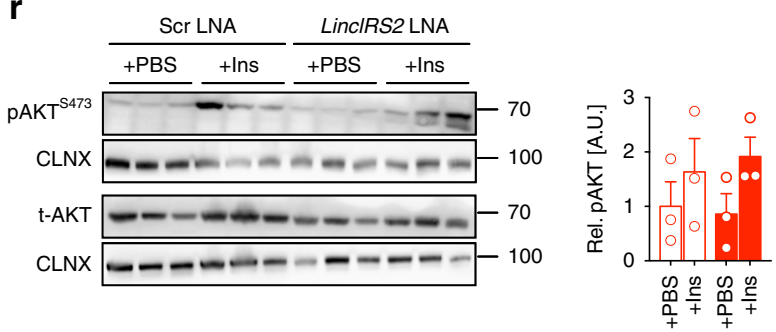

\section{RNA-Sequencing data processing.}

(1) Mouse RNA-Seq was processed utilizing the GRCm38 assembly of the mouse genome as gene sets from Ensembl release $90^{60}$. Biotype and gene features were added manually using Ensembl Biomart. The pipeline consists of six steps: (i) barcode and adapter removal using flexbar $3.4 .0^{61}$, (ii) computational rRNA depletion by filtering reads that map to known rRNAs in mice using Bowtie2 2.2.962, (iii) alignment of non-rRNA reads to the

mm10 reference genome using STAR 2.6.0 $\mathrm{c}^{63}$, (iv) transcript assembly using cufflinks followed by (v) cuffmerge, and (vi) cuffdiff performs DGE analysis between experimental conditions via Cufflinks suite 2.2.164.

(2) Human RNA-Seq data were analyzed using QuickNGS ${ }^{65}$, version 1.2.2 based on Ensembl release 82. In brief, reads were mapped to GRCm38 assembly of the human genome using Tophat $2^{66}$, version 2.0 .10 , and reassembled with Cufflinks, version 2.1.1. DGE was analyzed using the DESeq $2^{67}$, version 1.10.1. The results were uploaded to the QuickNGS 
Fig. 5 Knockdown of MAFG-dependent LincIRS2 causes mild insulin resistance in lean mice. a qPCR of LinclRS2 expression in the liver from C57BL/6N mice treated with Scr LNA $(n=7)$, Mafg LNA $(n=8)$, Control ASO $(n=10)$, or Mafg ASO $(n=7)$. b-f qPCR for LinclRS2 expression in primary hepatocytes after $\mathbf{b} 24 \mathrm{~h}$ of transduction with adenoviruses (Ad)-expressing caChREBP and caFOXO1, c $16 \mathrm{~h}$ of $200 \mathrm{nM} \mathrm{GCG}$ and $100 \mu \mathrm{M}$ cAMP, d $6 \mathrm{~h}$ of $27.5 \mathrm{mM}$ glucose, and/or e $100 \mathrm{nM}$ insulin in livers from mice with hepatocyte-specific IR knockout vs. Cre-negative Controls ${ }^{59}(n=3)$. f Twenty-four hours after transduction of primary hepatocytes with Ad-caSREBP1c. $\mathbf{g}$ qPCR of LincIRS2 expression after transfection of primary hepatocytes with $100 \mathrm{nM}$ LincIRS2 or Scr LNAs and $6 \mathbf{h}$ of stimulation with $\mathbf{g}$ FD and GCG or $\mathbf{h}$ insulin. $\mathbf{g}, \mathbf{h} n=6-10$ independent experiments, each in triplicate. i-k qPCR of Pck1 expression after transfection with $100 \mathrm{nM}$ LincIRS2 or Scr LNAs and $6 \mathrm{~h}$ of stimulation with $\mathbf{i}$ insulin, $\mathbf{j}$ FD, or $\mathbf{k}$ GCG. $\mathbf{i}-\mathbf{k} n=5-10$ experiments, each in triplicates. I qPCR of LincIRS2 expression in indicated tissues from 21-week-old CD-fed mice after 15 weeks of $10 \mathrm{mg} \mathrm{kg}^{-1}$ LinclRS2 or Scr LNA ( $\left.n=4\right)$ treatment. $\mathbf{m}$, $\mathbf{n}$ Body weight in $\mathbf{m}$ CD- and $\mathbf{n}$ HFD-fed C57BL/6N mice during 15 weeks of LincIRS2 LNA or Scr LNA $(n=8)$ treatment. $\mathbf{o}$ Fasted and random-fed GTT and ITT in CD-fed 12- and 11-week-old C57BL/6N mice after 6 and 5 weeks of LincIRS2 or Scr LNA $(n=8)$ treatment. $\mathbf{p}$ Fasted and random-fed glucose and ITT in HFD-fed 12- and 11-week-old C57BL/6N mice after 6 and 5 weeks of LincIRS2 or Scr LNA ( $n=8$ each) treatment. q, $\mathbf{r}$ Representative immunoblot and densitometry of t-AKT and pAKT 473 in $\mathbf{p}$ CD- or $\mathbf{r}$ HFD-fed C57BL/6N mice after 15 weeks of Linc/RS2 or Scr LNA treatment injected with $2.5 \mu$ l $g^{-1}$ BW $\mathrm{NaCl} 0.9 \%$ or $0.1 \mathrm{U} /$ mouse insulin $(n=3)$. Bar graphs represent mean \pm s.e.m. with all data plotted and statistical differences calculated using $(\mathbf{a}-\mathbf{i})$, I unpaired, two-tailed Student's $t$ test. q One-way ANOVA or $\mathbf{m}$-p two-way ANOVA with Bonferroni post correction for multiple testing. ${ }^{\star} p<0.05,{ }^{\star \star} p<$ $0.01,{ }^{* \star *} p<0.001$. Source data are provided as a Source Data file.

database and combined with multiple annotations using the biomaRt package version 2.16.0.

Gene overlap analysis. Overlap of regulated gene sets were performed with "venneuler" package for R using genes defined under (1) in the paragraph above. The $p$ value of the overlap was calculated using hypergeometric distribution

$$
p=\sum_{i=k}^{\min (m, n)} \frac{\left(\begin{array}{c}
m \\
i
\end{array}\right)\left(\begin{array}{c}
N-m \\
n-i
\end{array}\right)}{\left(\begin{array}{c}
N \\
n
\end{array}\right)}
$$

where $N$ is the total number of testable genes, $m$ is regulated genes in study A, $n$ is regulated genes in study $\mathrm{B}$, and $k$ is genes overlapping studies $\mathrm{A}$ and $\mathrm{B}$.

Ingenuity pathway analysis. IR, FOXO1, and SREBP1C/SREBF1 were loaded as seed gene nodes into IPA and expanded into TF-dependent networks by using the "grow" option with molecular interactions limited to "direct" and "downstream" effects. These manually curated pathways were overlaid with DGE information from RNA-Seq in HFD- vs. CD-fed C57BL/6N mice.

\section{Promoter analyses and motif enrichment analysis.}

(1) For enrichment analyses of known TFBS using $\mathrm{AME}^{33}$ functionality of MEME $^{34}$, RNA-Seq data of livers from mice on CD or HFD feeding were aligned to mm10 using $\mathrm{STAR}^{63}$, and transcriptome assembly and DGE analyses performed using CuffLinks/CuffDiff ${ }^{68}$, respectively. Significantly altered mRNAs and lncRNAs were defined by $p$ value $\leq 0.1$ and $\mathrm{L} 2 \mathrm{R}>0$ or $<0$. IncRNAs and mRNAs were defined by filtering for biotypes "bidirectional_promoter_lncRNA", "lincRNA", "sense_intronic", "sense_overlapping", "antisense", and "protein-coding" using annotations from Ensembl Biomart Version 90. Promoter sequences (defined as $-800 /+100$ bp from TSS) of each biotype class were extracted and enriched TFBS motifs identified using commands "ame-verbose 1-oc-bgformat 1-scoring avg-method ranksum-pvalue-report-threshold 0.05 db/JASPAR/JASPAR2018_CORE_vertebrates_non-redundant.meme db/MOUSE/uniprobe_mouse.meme".

(2) For de novo enrichment analyses of TFBS motifs using HOMER, mRNA, and IncRNA promoter sequences from (1) were used as input for HOMER de novo motif analyses (findMotifsGenome.pl) using indicated promoter lists as background. De novo motif analyses were collapsed to motifs known to bind TFs found expressed in at least one condition in the RNA-Seq data (CD/HFD), using the curated motif list from the IMAGE pipeline ${ }^{69}$.

Chromatin-immunoprecipitation (ChIP) sequencing analysis. Previously published public BLRP ChIP-seq data from livers of mice transduced with adenoviruses overexpressing BLRP-MAFG fusion protein (GEO ID: GSE77559) were used as proxy for MAFG cistromes in the liver. The ChIP-seq data were aligned to $\mathrm{mm} 10$ genome using STAR ${ }^{63}$, and BLRP-MAFG peaks were called in each replicate $(n=2)$ using findPeaks function in $\mathrm{HOMER}^{38}$. The peak lists were merged using mergePeaks function in HOMER and only peaks called in both replicates were retained for downstream analyses.

In vivo RNAi using LNAs. RNA-interference (RNAi)-mediated silencing of Mafg and LincIRS2 was performed using custom-made LNA GapmeRs that were designed and synthesized by Exiqon, Denmark to target murine Mafg and LincIRS2 (Mafg, LincIRS2 LNA). A negative control with no homology to known mouse transcripts was used as control LNA. Four-week-old C57BL/6N male mice were obtained from Charles River and acclimated for 2 weeks, followed by intravenous (i.v.) injection of $10 \mathrm{mg} \mathrm{kg}^{-1} \mathrm{BW}$ LNA dissolved in $0.9 \% \mathrm{NaCl}$ once every other week for 15 weeks starting from 6 weeks of age. One week after the start of injection series, mice were placed on CD or HFD for 14 weeks. Insulin and glucose tolerance test (ITTs and GTTs) were performed at 6 and 7 weeks of diet, respectively.

In vivo RNAi using antisense oligonucleotides (ASO). Six- to seven-week-old male C57BL/6N mice or Maff/Mafk-null (double knockout, DKO) mice were injected weekly with $5 \mathrm{mg} \mathrm{kg}^{-1} \mathrm{Gal}-\mathrm{Nac}$-conjugated Mafg or Control ASO at a concentration of $1 \mathrm{mg} / \mathrm{mL}$ dissolved in $1 \times$ phosphate-buffered saline (PBS), for 20 weeks. Two weeks after the first injection, mice were placed on HFD for 18 weeks. ITT was performed at 9 weeks on diet, and GTT was performed at 10 and 16 weeks on diet.

Generation of LincIRS2 ${ }^{\Delta / \Delta}$ knockout mice using CRISPR/Cas9. Generation of a mouse model for genetic LincIRS2 deficiency (LincIRS2 ${ }^{\Delta / \Delta}$ ) was performed using CRISPR/Cas9 genome editing where a 426-bp deletion (chr8:

$10,899,861-10,900,279)$ and a 5 -bp insertion, encompassing the presumed promoter region (defined by occurrence of histone 3 lysine 4 trimethylation (H3K4me3) near the LincIRS2 TSS in murine liver, based on NIH Epigenomics Roadmap information ${ }^{70}$ ) and exon 1 of LincIRS2 (GRCm38/mm10) were excised. To this end, guide RNAs (gRNAs, sequences provided in Supplementary Data 10) were designed using CRISPOR ${ }^{71}$ and gRNAs with highest specificity scores $(\geq 50)$ chosen, yet gRNAs with predicted off-target effects for 1 or 2 occurring mismatches excluded. After gRNA identification, target-specific CRISPR RNAs (crRNAs) were incubated with generic transactivating crRNAs (tracrRNAs, Integrated DNA Technologies) to form the active gRNA. Next, annealed gRNAs were incubated with Cas9 proteins to obtain functional ribonucleoprotein complexes. Pronuclear injections of CRISPR/Cas9 complexes into fertilized oocytes of C57BL/6N females were performed at the CECAD in vivo Research Facility ${ }^{2}$. Briefly, we employed pronuclear injection of CRISPR/Cas9 components into C57BL/6NRj zygotes, which were then implanted into pseudopregnant RjHan:NMRI females. Synthetic target-specific crRNA and sequence-independent tracrRNA molecules were purchased from commercial distributors. Shortly before injection, crRNAs and tracrRNAs were co-incubated with recombinant Cas9 proteins, and to enhance the efficacy of CRISPR/Cas9 genome editing, we added Cas9-encoding mRNA to the injection mix. Healthy, CRISPR/Cas9-injected oocytes were subsequently implanted into oviducts of pseudopregnant 0.5 postcoital foster females. Finally, offspring born from implanted oocytes was analyzed via DNA sequencing, and in the case of successful CRISPR/Cas9-mediated LincIRS2 knockout, bred to C57BL/6N mice to create C57BL/6-LincIRS2 ${ }^{\text {emlCecad }}$ transgenic mouse lines. Genotyping primers are available in Supplementary Data 10. For each of the two gRNAs, the top five sites of predicted off-target Cas9 activity were investigated using Sanger Sequencing, and no changes to the GRCm38/mm10 genomic sequence were detected (Supplementary Data 14).

Adeno-associated virus (AAV)-mediated MAFG overexpression. PscAAV8MAFG-HA encoding plasmids were produced by replacing luciferase (Luc) coding sequence in pscTTR-luc by the respective sequences. AAV8 was produced in HEK293 cells using the triple-transfection method. Specifically, the AAV helper plasmid pXR8, the adenoviral helper plasmid pXX6, and the vector plasmid (pscTTR-luc, pscAAV-MAFG-HA) were transfected in 1:1:1 molar ratio using $\mathrm{CaP}$ method. Forty-eight hours post transfection, cells were harvested and lysed. Following benzonase treatment and a low-speed centrifugation preclearing step, preparation was purified by iodixanol step gradient purification. Genomic titer was determined by qPCR using ITR-specific primers. C57BL/6N and LincIRS2 $\Delta / \Delta$ male 
a
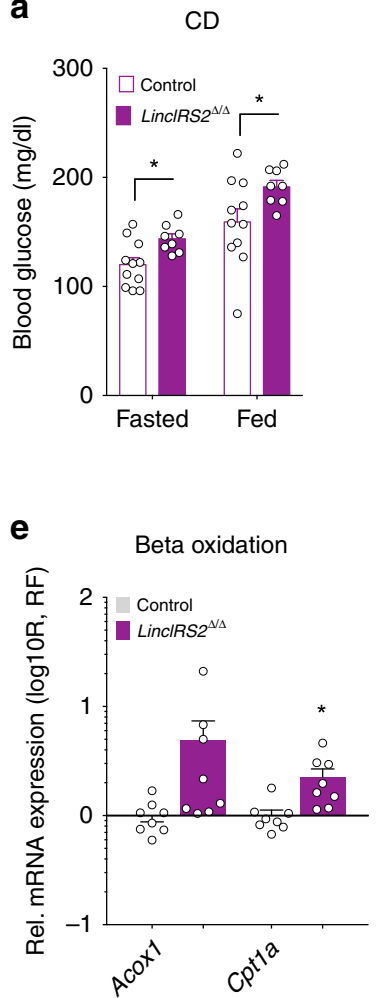

i

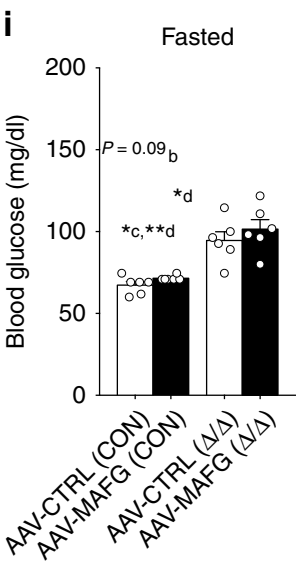

m

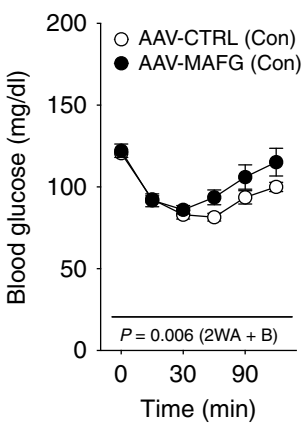

b

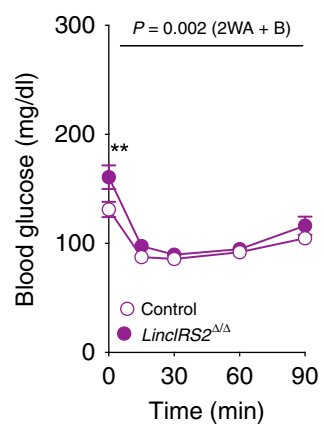

f

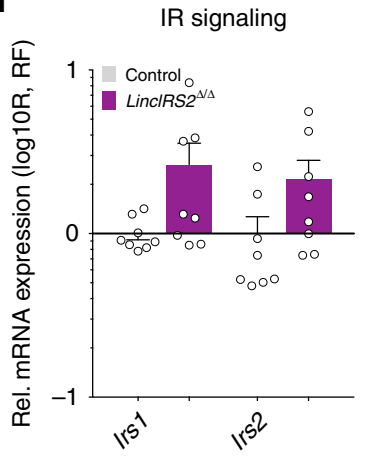

j

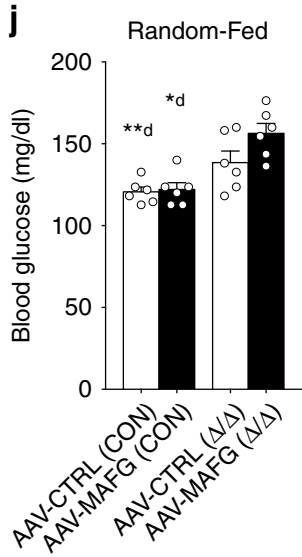

n

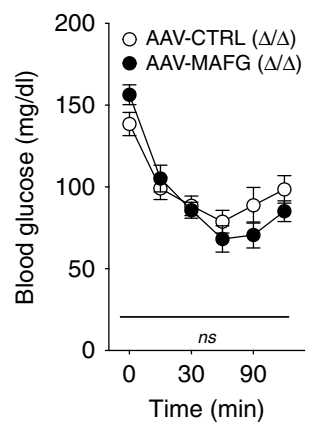

C

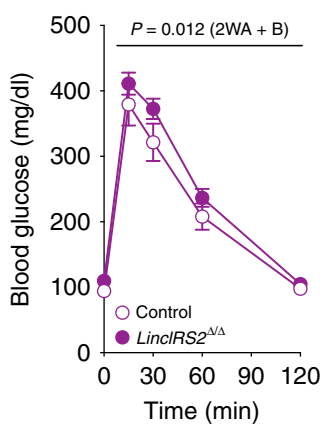

g

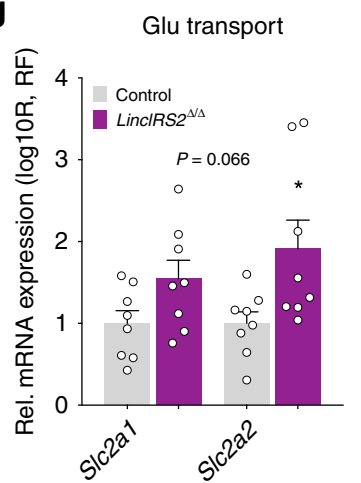

$\mathbf{k}$

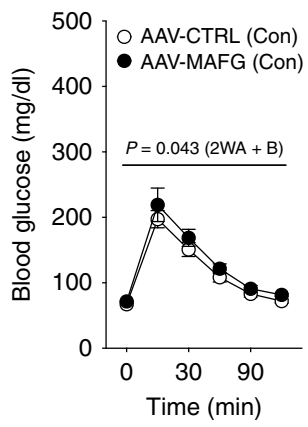

d Gluconeogenesis

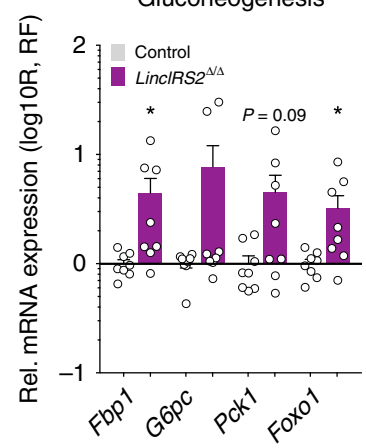

h
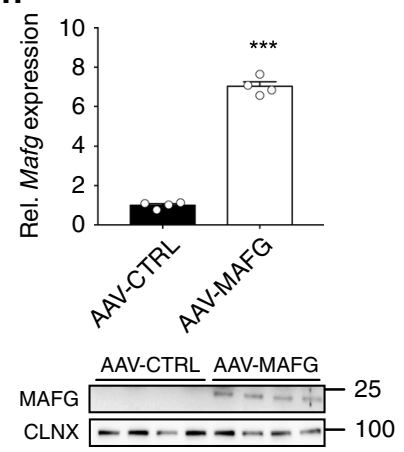

I

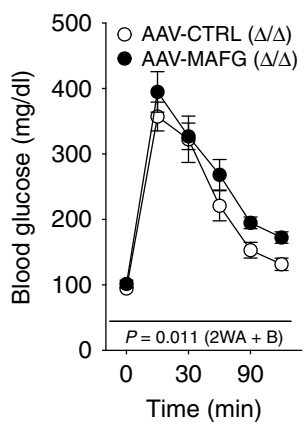

o

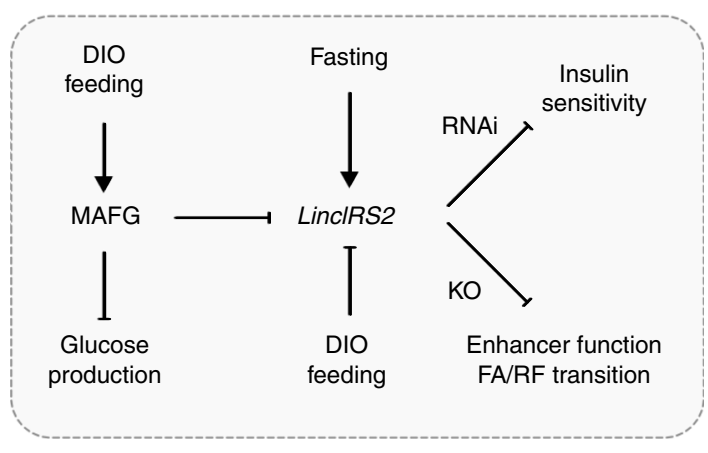

mice were 8-10 weeks of age and i.v. injected with $10 \mathrm{E}^{11}$ vector particles/animal of genomic copies for AAV control (encoding Luc) or AAV-MAFG. Glucose and insulin tolerance test were performed at 8 and 9 weeks after injection.

Isolation of primary hepatocytes and liver fractionation. Mouse primary hepatocytes were isolated from C57BL/6N mice livers by a classical two-step collagenase perfusion method ${ }^{73}$ with minor modifications as follows: mice were subjected to experiments around 8-12 weeks of age. Under anesthesia, mice were perfused via the portal vein with $50 \mathrm{ml}$ of perfusion medium, followed by digestion with $50 \mathrm{ml}$ of collagenase medium. After liver perfusion, dissociated cells from the liver were filtered through a 70- $\mu \mathrm{m}$ cell filter (BD Falcon) into DMEM, low glucose (Gibco) supplemented with $10 \%$ FBS and $1 \%$ penicillin-streptomycin (P/S), and centrifuged twice at $50 \mathrm{~g}$ for $3 \mathrm{~min}$ to recover the pellet and the supernatant. 
Fig. 6 CRISPR-mediated deletion of LincIRS2 impairs glucose metabolism in lean mice. a Fasted and random-fed glucose in CD-fed 12-week-old LincIRS2 ${ }^{\Delta / \Delta}$ mutant mice $(n=8)$ vs. wild-type littermates $(n=11)$. b ITT in 11-week-old and $\mathbf{c}$ GTT in 12 -week-old, CD-fed LinclRS2 ${ }^{\Delta / \Delta}(n=8)$ vs. Control mice $(n=11)$. $\mathbf{d}-\mathbf{g}$ qPCR of $\mathbf{d}$ gluconeogenic $\mathbf{e}$ oxidative $\mathbf{f} I R$ signaling and $\mathbf{g}$ glucose transporter expression in 8-15-week-old LinclRS2 $4 / \Delta$ vs. LinclRS2 $4 / w t$ $(n=8)$ mice. $\mathbf{h}$ Hepatic qPCR and immunoblot $(n=4)$ from 18- to 20 -week-old C57BL/6N 10 weeks after i.v. injection with $1 \times 10 E 11$ genomic copies/ animal encoding AAV8-CTRL or AAV8-MAFG. Fasted $\mathbf{i}$ and random-fed $\mathbf{j}$ glucose. $\mathbf{k}, \mathbf{I}$ GTT and $\mathbf{m}, \mathbf{n}$ ITT in CD-fed 18-20-week-old LinclRS2 $4 / \Delta$ vs. C57BL/ $6 \mathrm{~N}$ Controls $(n=12$ each) with half of mice in each genotype $(n=6)$ i.v. injected with $1 \times 10$ E11 genomic copies/animal AAV8-CTRL or AAV8-MAFG. - Illustration of MAFG and LincIRS2 in regulation of hepatic glucose metabolism. Bar graphs represent mean \pm s.e.m. with all data plotted and statistical differences were calculated using $\mathbf{a}, \mathbf{d}-\mathbf{h}$ unpaired, two-tailed Student's $t$ test. $\mathbf{i}, \mathbf{j}$ One-way ANOVA with Bonferroni post correction for multiple testing. b, c, k-n Two-way ANOVA with Bonferroni post correction for multiple testing. Superscripts depict group comparisons for post analysis (a-f $=$ comparison vs. columns 1-6) ${ }^{\star} p<0.05,{ }^{\star \star} p<0.01,{ }^{\star \star \star} p<0.001$. Source data are provided as a Source Data file.

Hepatocytes were obtained by resuspending the pellet in 30\% Percoll and centrifuging at $150 \mathrm{~g}$ for $7 \mathrm{~min}$. For obtaining non-hepatocyte (non-parenchymal) cell fractions, the supernatant was centrifuged at $350 \mathrm{~g}$, and the resulting cell pellets centrifuged on a $20 \%$ (w/vol) Histodenz (Sigma) gradient. For fractionation analyses, both hepatocytes and non-parenchymal fractions were frozen immediately after isolation. For experiments in which hepatocytes were transfected and/or stimulated, cells were attached to six-well collagen I-coated plates (Costar) for $2 \mathrm{~h}$ and were grown in P/S-supplemented DMEM, low glucose (Gibco) without FBS overnight; they were then subjected to experimental procedures $24 \mathrm{~h}$ after isolation. The media were as follows: Perfusion medium, HBSS (Gibco) without magnesium or calcium and supplemented with $0.5 \mathrm{mM}$ EGTA; collagenase medium, DMEM, low glucose (Gibco) supplemented with $15 \mathrm{mM}$ HEPES and 100 collagen digestion units (CDU) $\mathrm{ml}^{-1}$ of collagenase, type IV (Worthington); $90 \%$ concentrated Percoll, 100\% Percoll (Amersham) diluted with 10× HBSS (Gibco).

Subcellular fractionation of primary hepatocytes. Subcellular fractions were obtained using freshly isolated primary hepatocytes using Nuclei Isolation Kits: Nuclei EZ Prep according to the manufacturer's (Sigma Aldrich) protocol. Briefly, $5 \times 10^{6}$ cells were centrifuged $\left(5 \mathrm{~min}, 300 \mathrm{~g}, 4^{\circ} \mathrm{C}\right.$ ) and supernatants were discarded, whereas cell pellets were resuspended in $10 \mathrm{ml}$ of PBS and centrifuged again $(5 \mathrm{~min}$, $300 \mathrm{~g}, 4^{\circ} \mathrm{C}$ ). After removal of supernatants, $4 \mathrm{ml}$ of ice-cold Nuclei EZ Lysis Buffer was added to cell pellets and the reaction tube vortexed briefly and kept on ice for $5 \mathrm{~min}$. Following centrifugation $\left(5 \mathrm{~min}, 300 \mathrm{~g}, 4^{\circ} \mathrm{C}\right)$, supernatants were kept for later analysis (cytoplasmic fraction), while pellets were resuspended in $4 \mathrm{ml}$ of icecold Nuclei EZ Lysis Buffer, vortexed briefly, and kept on ice for 5 min. After a final centrifugation $\left(5 \mathrm{~min}, 300 \mathrm{~g}, 4^{\circ} \mathrm{C}\right)$ supernatants were discarded and nuclei pellets resuspended in $200 \mu \mathrm{l}$ of ice-cold Nuclei EZ Storage Buffer (nuclear fraction). If not directly processed for RNA isolation, cytoplasmic and nuclear fractions were stored at $-80^{\circ} \mathrm{C}$

LNA-mediated gene knockdown of primary hepatocytes. Primary hepatocytes from C57BL/6N mice were cultured for $24 \mathrm{~h}$ at $37^{\circ} \mathrm{C}$ and $5 \% \mathrm{CO}_{2}$ before transfection of control (Scr), Mafg, and LincIRS2 (4833411C07Rik) LNAs (sequences provided in Supplementary Data 10). Lipofectamine RNAiMax was diluted 1:16 in DMEM. For a final concentration of $100 \mathrm{nM}$, the respective LNAs (stock $10 \mu \mathrm{M}$ ) were diluted 1:100 in DMEM. Both solutions were incubated for $5 \mathrm{~min}$ at room temperature. LNA and Lipofectamine solutions were mixed at equal volumes and incubated for $5 \mathrm{~min}$. Cells were washed with prewarmed $1 \times \mathrm{PBS}$ and $1.6 \mathrm{ml}$ of DMEM without FBS added to each well. Four-hundred microliters of LNA/Lipofectamine mix was added and cells incubated for $24 \mathrm{~h}$ at $37^{\circ} \mathrm{C}$ and $5 \% \mathrm{CO}_{2}$ before changing the medium. For in vitro stimulation experiments, primary hepatocytes were washed with prewarmed $1 \times$ PBS and fresh medium added. Cells were stimulated with $100 \mathrm{nM}$ Insulin (Sigma), Glucagon $\left(0.1 \mathrm{mg} \mathrm{ml}^{-1}\right.$, Sigma), $10 \mu \mathrm{M}$ Forskolin plus $100 \mathrm{nM}$ Dexamethasone (FD), or FD plus $100 \mathrm{nM}$ Insulin (FDI), and incubated for $10 \mathrm{~min}, 6 \mathrm{~h}$, or $24 \mathrm{~h}$ at $37^{\circ} \mathrm{C}$ and $5 \% \mathrm{CO}_{2}$, before harvesting cells for further experimental analysis.

Quantification of glucose production. After $24 \mathrm{~h}$ of transfection, primary hepatocytes were washed with warm $1 \times$ PBS, and glucose-free DMEM (Gibco) supplemented with $100 \mathrm{mM}$ sodium pyruvate was added in order to study cellintrinsical glucose production. Stimulation with Fsk/Dex $(10 \mu \mathrm{M} / 100 \mathrm{nM})$ with/ without $100 \mathrm{nM}$ insulin was performed for $24 \mathrm{~h}$ at $37^{\circ} \mathrm{C}$ and $5 \% \mathrm{CO}_{2}$. Supernatant medium of the cells was collected to measure endogenous glucose production with Glucose-Glo Assay Kits (Promega) following the manufacturer's instructions.

\section{Adenoviral overexpression of metabolic TF in hepatocytes. Adenoviruses} encoding YFP (Addgene plasmid \#15302), constitutively active mouse FOXO1 (caFOXO1, Addgene plasmid \#17547), and constitutively active mouse SREBP1c (caSREBP1C, Addgene plasmid \#8883) were subcloned from plasmids (Addgene) into the adenoviral vector system $\mathrm{pAd} / \mathrm{CMV} / \mathrm{V} 5-\mathrm{DEST}^{\mathrm{m}}$ (Invitrogen). A constitutively active mouse ChREBP lacking the $\mathrm{N}$-terminal low-glucose inhibitory domain was PCR amplified from mouse liver $\mathrm{cDNA}^{74}$ and cloned into pAd/CMV/ V5-DEST. Adenoviruses were amplified in HEK293A cells and purified by $\mathrm{CsCl}$ gradient centrifugation. Purified viruses were desalted with PD10 columns (GE
Healthcare Life Sciences) and were titered with an Adeno-X Rapid Titer Kit (Clontech). Primary hepatocytes were treated with adenoviruses encoding aforementioned TF for indicated times. For in vitro stimulation experiments, cells were exposed to $100 \mu \mathrm{M}$ cAMP or $200 \mathrm{nM}$ GCG and incubated for $6 \mathrm{~h}$ or stimulated with $100 \mathrm{nM}$ Insulin (Sigma) for $16 \mathrm{~h}$, at $37^{\circ} \mathrm{C}$ and $5 \% \mathrm{CO}_{2}$, before harvesting cells for experimental analysis.

Immunoblot analysis. For protein isolation, primary hepatocytes grown on sixwell plates were washed gently one time with ice-cold $1 \times$ PBS and excess $1 \times$ PBS aspirated. Five-hundred microliters of RIPA lysis buffer (50 mM Tris-HCL, pH 7.5, $150 \mathrm{mM} \mathrm{NaCl}, 1 \mathrm{mM}$ EDTA, $0.1 \%$ sodium deoxycholate, $1 \% \mathrm{NP}-40,1 \times$ protease inhibitor (Sigma), and $1 \times$ phosphatase inhibitor (Roche)) was added to each well of the six-well plate and cells were scraped. Protein lysates were transferred to $1.5-\mathrm{ml}$ Eppendorf tubes, snap-frozen in liquid nitrogen, and thawed on ice for three repeated cycles. Tissues were homogenized in $1 \mathrm{ml}$ of RIPA Buffer using FastPrep $24 \mathrm{G}$ (MPBio). After $10 \mathrm{~min}$ of centrifugation at $12,000 \mathrm{~g}$ and $4^{\circ} \mathrm{C}$, the supernatant was collected into fresh tubes, and protein concentration was determined using the Bradford colorimetric protein method (Thermo Fisher Scientific). Protein samples were stored at $-80^{\circ} \mathrm{C}$. Samples for immunoblotting were separated by sodium dodecyl sulfate polyacrylamide gel electrophoresis after being prepared with $4 \times$ Laemmli Sample Buffer containing $10 \% \beta$-mercaptoethanol and heated to $95^{\circ} \mathrm{C}$ for $5 \mathrm{~min}$. Subsequently, proteins were transferred to nitrocellulose membrane and blocked with Western Blocking Reagent (Roche). Membranes were incubated with primary antibodies at $4{ }^{\circ} \mathrm{C}$ overnight. After incubation with secondary antibodies, blots were developed with Pierce ECL Western Blotting substrate (Thermo Fisher Scientific). All densiometric measurements were performed with ImageJ. Band intensity was normalized to calnexin (CLNX), which served as internal loading control, blotted on the same gel and membrane. Antibodies were purchased from Cell Signaling Technology and raised against $\mathrm{pAKT} \mathrm{S}^{\mathrm{S} 73}$ (Catalog No. 9271), t-AKT

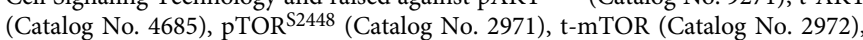
p4E-BP1 ${ }^{\text {T37/46 }}$ (Catalog No. 2855), t-4E-BP1 (Catalog No. 9452), pGSK3B ${ }^{\text {S9 }}$ (Catalog No. 9323), t-GSK3B (Catalog No. 9315), and NRF1/NFE2L1 (Catalog No 8052). MAFG antibody was purchased from GeneTex (GTX 114541). Primary antibodies were used at a 1:1000 dilution ratio in Tween TBS. CLNX antibody was used at a 1:5,000 dilution and purchased from Calbiochem (Catalog No. 208-880).

Glucose, insulin, and PTT. At the time of performing insulin tolerance tests (ITT), mice were 11 weeks of age and either 4 weeks exposed to CD or HFD feeding. ITT was carried out in random-fed mice in the morning. After determining basal blood glucose levels, each animal received $0.75 \mathrm{IU}$ per kg BW of insulin (Insuman Rapid; Sanofi-Aventis). For glucose tolerance tests (GTT), mice were 12 weeks of age and 5 weeks exposed to CD or HFD feeding. After $16 \mathrm{~h}$ of fasting and measurement of basal blood glucose levels, animals were i.p. injected with $2 \mathrm{~g}$ per kg BW of glucose (20\% glucose, Delta select). Blood glucose levels were recorded using an automatic glucose monitor (Contour, Bayer Diabetes Care) at the indicated time points in (1) male Scr vs. Mafg and LincIRS2 LNA-treated mice, (2) Control ASO vs. Mafg ASO, and (3) LincIRS2 ${ }^{\mathrm{wt}} / \mathrm{wt}$ (Control for LincIRS2 ${ }^{\Delta / \Delta}$ ) and LincIRS2 ${ }^{\Delta / \Delta}$ mice. In the ASO cohort, animals that showed no decrease of blood glucose after insulin gavage throughout all measurements were excluded from ITT analysis, assuming injection of insulin outside of the peritoneal cavity as required for the assay. The pyruvate tolerance test (PTT) was performed in HFD-fed animals after 6 weeks of HFD feeding with Scr and Mafg LNA injection 5 days before the experiment. For the PTT itself, $4 \mathrm{~g}$ of pyruvate was dissolved in $20 \mathrm{ml}$ of $0.9 \% \mathrm{NaCl}$ and injected $10 \mu \mathrm{g} \mathrm{g}^{-1} \mathrm{BW}$ after $16 \mathrm{~h}$ of fasting (final dose $2 \mathrm{~g}$ pyruvate $\mathrm{kg}^{-1}$ ).

Indirect calorimetry (PhenoMaster). Upon indirect calorimetry measurements, mice of indicated genotypes and diets were 16 weeks of age and 10 weeks exposed to CD or HFD. All measurements were performed using a PhenoMaster System (TSE Systems), which allowed in-cage metabolic and activity monitoring. Three days before analysis, mice were placed alone in training cages, identical to the 7.1-1 chambers of the PhenoMaster open-circuit calorimetry system and continued to receive the respective diets $(C D$ and $H F D)$ throughout training and data acquisition. Diets and water were provided AL in the appropriate devices, and food intake measured by the built-in automated instruments. Parameters of indirect 
calorimetry were measured initially for $96 \mathrm{~h}$ at $22^{\circ} \mathrm{C}$ and mean values calculated for each time of day.

Tissue collection. At the end of the experimental protocol, LNA-injected mice were randomly assigned to either intravenous (i.v.) injection of $0.9 \% \mathrm{NaCl}$ or $0.1 \mathrm{U} /$ animal of insulin (Sigma). All mice were sacrificed by carbon dioxide $\left(\mathrm{CO}_{2}\right)$ asphyxiation. All tissues were washed with $1 \times$ PBS, weighed, snap-frozen in liquid nitrogen, and stored at $-80^{\circ} \mathrm{C}$.

Enzyme-linked immunosorbent assay. Fibroblast Growth Factor 21 Mouse (FGF21) ELISA was obtained from Biovendor RD291108200R (Czech Republic), and performed using serum from CD and HFD-fed mice, Scr vs. Mafg LNA treated following the manufacturer's instructions.

Histological staining. For immunostaining, liver tissues were stored in PFA $4 \%$ for $24 \mathrm{~h}$ and then $70 \%$ ethanol for histological analysis. Transveral cryosections from paraffin-embedded liver were prepared, fixed, and stained by hematoxylin and $\operatorname{eosin}(\mathrm{H} / \mathrm{E})$

RNA isolation and quantitative RT-PCR (qPCR) analysis. Total RNA was isolated from primary hepatocytes and whole tissues using peqGOLD TriFast (PEQ LAB Biotechnologie). mRNA was reverse transcribed into complementary DNA using High Capacity cDNA reverse transcription kit (Applied Biosystems). Abun dances of mRNAs were quantified by TaqMan Assay on Demand Kits (Applied Biosystems) according to the manufacturer's protocol if not indicated otherwise. Abundances of lncRNAs were quantified using SYBR methodology using Select Master Mixes (Thermo Fisher). The relative abundance of mRNAs was calculated using comparative methods $\left(2^{-\Delta \Delta C T}\right)$ according to ABI Relative Quantification Methods. Transcript levels were normalized to hypoxanthine phosphoribosyl transferase 1 (Hprt1) or glyceraldehyde-3-phosphate dehydrogenase (Gapdh) expression; Hprt and Gapdh abundances remained unaffected across experimental conditions. SYBR primer sequences are provided in Supplementary Data 10.

Reporting summary. Further information on research design is available in the Nature Research Reporting Summary linked to this article.

\section{Data availability}

The source data underlying Fig. 1c-i, Fig. 2b, d, e, g, i, Fig. 3a-h, Fig. 4a-j, l-r, Fig. 5a-r, Fig. a-n, and Supplementary Fig. 1d-f, h-I, Fig. 2c-k, Fig. 3a-g, i-j, Fig. 4b, k-n, Fig. $5 \mathrm{a}-\mathrm{b}$, e are provided as Source Data file. Data from RNA-Seq are available under GEO Super Series GSE121346.

Received: 23 October 2018; Accepted: 27 December 2019; Published online: 31 January 2020

\section{References}

1. Rui, L. Energy metabolism in the liver. Compr. Physiol. 4, 177-197 (2014).

2. Lempradl, A., Pospisilik, J. A. \& Penninger, J. M. Exploring the emerging complexity in transcriptional regulation of energy homeostasis. Nat. Rev. Genet. 16, 665-681 (2015).

3. Van Gaal, L. F., Mertens, I. L. \& De Block, C. E. Mechanisms linking obesity with cardiovascular disease. Nature 444, 875-880 (2006).

4. Hotamisligil, G. S. Endoplasmic reticulum stress and atherosclerosis. Nat. Med. 16, 396-399 (2010).

5. Torres, D. M., Williams, C. D. \& Harrison, S. A. Features, diagnosis, and treatment of nonalcoholic fatty liver disease. Clin. Gastroenterol. Hepatol. 10, 837-858 (2012).

6. Birney, E. et al. Identification and analysis of functional elements in $1 \%$ of the human genome by the ENCODE pilot project. Nature 447, 799-816 (2007).

7. Carninci, P. et al. The transcriptional landscape of the mammalian genome. Science 309, 1559-1563 (2005)

8. Zhao, Y., Yuan, J. \& Chen, R. NONCODEv4: annotation of noncoding RNAs with emphasis on long noncoding RNAs. Methods Mol. Biol. 1402, 243-254 (2016).

9. Mattick, J. S. \& Rinn, J. L. Discovery and annotation of long noncoding RNAs. Nat. Struct. Mol. Biol. 22, 5-7 (2015).

10. Clark, M. B. et al. The reality of pervasive transcription. PLoS Biol. 9, e1000625 (2011). discussion e1001102.

11. Derrien, T. et al. The GENCODE v7 catalog of human long noncoding RNAs: analysis of their gene structure, evolution, and expression. Genome Res. 22, 1775-1789 (2012).

12. Ulitsky, I. \& Bartel, D. P. lincRNAs: genomics, evolution, and mechanisms. Cell 154, 26-46 (2013).
13. Iyer, M. K. et al. The landscape of long noncoding RNAs in the human transcriptome. Nat. Genet. 47, 199-208 (2015).

14. Kong, L. et al. CPC: assess the protein-coding potential of transcripts using sequence features and support vector machine. Nucleic Acids Res. 35, W345-W349 (2007)

15. Tichon, A. et al. A conserved abundant cytoplasmic long noncoding RNA modulates repression by Pumilio proteins in human cells. Nat. Commun. 7, 12209 (2016).

16. Rinn, J. L. et al. Functional demarcation of active and silent chromatin domains in human HOX loci by noncoding RNAs. Cell 129, 1311-1323 (2007).

17. Mele, M. \& Rinn, J. L. "Cat's Cradling” the 3D genome by the act of LncRNA transcription. Mol. Cell 62, 657-664 (2016).

18. Khalil, A. M. et al. Many human large intergenic noncoding RNAs associate with chromatin-modifying complexes and affect gene expression. Proc. Natl Acad. Sci. USA 106, 11667-11672 (2009).

19. Mondal, T. et al. MEG3 long noncoding RNA regulates the TGF-beta pathway genes through formation of RNA-DNA triplex structures. Nat. Commun. 6, 7743 (2015).

20. Lin, A. et al. The LINK-A IncRNA interacts with PtdIns $(3,4,5) \mathrm{P} 3$ to hyperactivate AKT and confer resistance to AKT inhibitors. Nat. Cell Biol. 19, 238-251 (2017).

21. Kleaveland, B., Shi, C. Y., Stefano, J. \& Bartel, D. P. A network of noncoding regulatory RNAs acts in the mammalian brain. Cell 174, 350-362 (2018).

22. Sallam, T. et al. Feedback modulation of cholesterol metabolism by the lipidresponsive non-coding RNA LeXis. Nature 534, 124-128 (2016)

23. Yang, L. et al. IncRNA-dependent mechanisms of androgen-receptorregulated gene activation programs. Nature 500, 598-602 (2013).

24. Li, P. et al. A liver-enriched long non-coding RNA, lncLSTR, regulates systemic lipid metabolism in mice. Cell Metab. 21, 455-467 (2015).

25. Chen, G. et al. LncRNA SRA promotes hepatic steatosis through repressing the expression of adipose triglyceride lipase (ATGL). Sci. Rep. 6, 35531 (2016).

26. Ruan, X., Li, P., Cangelosi, A., Yang, L. \& Cao, H. A Long Non-coding RNA, IncLGR, Regulates Hepatic Glucokinase Expression and Glycogen Storage during Fasting. Cell Rep. 14, 1867-1875 (2016).

27. Zhu, X., Wu, Y. B., Zhou, J. \& Kang, D. M. Upregulation of lncRNA MEG3 promotes hepatic insulin resistance via increasing FoxO1 expression. Biochem. Biophys. Res. Commun. 469, 319-325 (2016).

28. Kinsella, R. J. et al. Ensembl BioMarts: a hub for data retrieval across taxonomic space. Database 2011, bar030 (2011)

29. Eissing, L. et al. De novo lipogenesis in human fat and liver is linked to ChREBP-beta and metabolic health. Nat. Commun. 4, 1528 (2013).

30. Wang, L. et al. CPAT: Coding-Potential Assessment Tool using an alignmentfree logistic regression model. Nucleic Acids Res. 41, e74 (2013).

31. Alam, T. et al. Promoter analysis reveals globally differential regulation of human long non-coding RNA and protein-coding genes. PloS One 9, e109443 (2014).

32. Mele, M. et al. Chromatin environment, transcriptional regulation, and splicing distinguish lincRNAs and mRNAs. Genome Res. 27, 27-37 (2016).

33. Buske, F. A., Boden, M., Bauer, D. C. \& Bailey, T. L. Assigning roles to DNA regulatory motifs using comparative genomics. Bioinformatics 26, 860-866 (2010).

34. Bailey, T. L., Johnson, J., Grant, C. E. \& Noble, W. S. The MEME Suite. Nucleic Acids Res. 43, W39-W49 (2015).

35. Qi, M. et al. Analysis of long non-coding RNA expression of lymphatic endothelial cells in response to type 2 diabetes. Cell Physiol. Biochem. 41, 466-474 (2017).

36. Marini, M. G. et al. hMAF, a small human transcription factor that heterodimerizes specifically with Nrf1 and Nrf2. J. Biol. Chem. 272, 16490-16497 (1997)

37. Johnsen, O., Murphy, P., Prydz, H. \& Kolsto, A. B. Interaction of the CNCbZIP factor TCF11/LCR-F1/Nrf1 with MafG: binding-site selection and regulation of transcription. Nucleic Acids Res. 26, 512-520 (1998).

38. Heinz, S. et al. Simple combinations of lineage-determining transcription factors prime cis-regulatory elements required for macrophage and B cell identities. Mol. Cell 38, 576-589 (2010).

39. Yamazaki, H., Katsuoka, F., Motohashi, H., Engel, J. D. \& Yamamoto, M. Embryonic lethality and fetal liver apoptosis in mice lacking all three small Maf proteins. Mol. Cell. Biol. 32, 808-816 (2012).

40. de Aguiar Vallim, T. Q. et al. MAFG is a transcriptional repressor of bile acid synthesis and metabolism. Cell Metab. 21, 298-310 (2015).

41. Balwierz, P. J. et al. ISMARA: automated modeling of genomic signals as a democracy of regulatory motifs. Genome Res. 24, 869-884 (2014).

42. Yang, Y. \& Cvekl, A. Large Maf transcription factors: cousins of AP-1 proteins and important regulators of cellular differentiation. Einstein J. Biol. Med. 23, $2-11(2007)$ 
43. Hirotsu, Y. et al. Nrf2-MafG heterodimers contribute globally to antioxidant and metabolic networks. Nucleic Acids Res. 40, 10228-10239 (2012).

44. Katsuoka, F., Yamazaki, H. \& Yamamoto, M. Small Maf deficiency recapitulates the liver phenotypes of Nrf1- and Nrf2-deficient mice. Genes Cells 21, 1309-1319 (2016).

45. Yang, L. et al. Integrative transcriptome analyses of metabolic responses in mice define pivotal LncRNA metabolic regulators. Cell Metab. 24, 627-639 (2016).

46. Patel, S. et al. Tissue-specific role of glycogen synthase kinase 3beta in glucose homeostasis and insulin action. Mol. Cell. Biol. 28, 6314-6328 (2008).

47. Laplante, M. \& Sabatini, D. M. mTOR signaling in growth control and disease. Cell 149, 274-293 (2012).

48. Sonenberg, N. \& Hinnebusch, A. G. Regulation of translation initiation in eukaryotes: mechanisms and biological targets. Cell 136, 731-745 (2009).

49. Dennis, M. D., Jefferson, L. S. \& Kimball, S. R. Role of p70S6K1-mediated phosphorylation of eIF4B and PDCD4 proteins in the regulation of protein synthesis. J. Biol. Chem. 287, 42890-42899 (2012).

50. Jimenez, V. et al. FGF21 gene therapy as treatment for obesity and insulin resistance. EMBO Mol. Med. 10, e8791 (2018).

51. Kotkow, K. J. \& Orkin, S. H. Complexity of the erythroid transcription factor NF-E2 as revealed by gene targeting of the mouse p18 NF-E2 locus. Proc. Natl Acad. Sci. USA 93, 3514-3518 (1996).

52. Uyeda, K. \& Repa, J. J. Carbohydrate response element binding protein, ChREBP, a transcription factor coupling hepatic glucose utilization and lipid synthesis. Cell Metab. 4, 107-110 (2006).

53. Gross, D. N., Wan, M. \& Birnbaum, M. J. The role of FOXO in the regulation of metabolism. Curr. Diab. Rep. 9, 208-214 (2009).

54. Horton, J. D., Goldstein, J. L. \& Brown, M. S. SREBPs: activators of the complete program of cholesterol and fatty acid synthesis in the liver. J. Clin. Investig. 109, 1125-1131 (2002).

55. Hezroni, H. et al. Principles of long noncoding RNA evolution derived from direct comparison of transcriptomes in 17 species. Cell Rep. 11, 1110-1122 (2015).

56. Diederichs, $\mathrm{S}$. The four dimensions of noncoding RNA conservation. Trends Genet. 30, 121-123 (2014)

57. Roux, B. T., Heward, J. A., Donnelly, L. E., Jones, S. W. \& Lindsay, M. A. Catalog of differentially expressed long non-coding RNA following activation of human and mouse innate immune response. Front. Immunol. 8, 1038 (2017).

58. Widenmaier, S. B. et al. NRF1 Is an ER membrane sensor that is central to cholesterol homeostasis. Cell 171, 1094-1109 e1015 (2017).

59. Okada, T. et al. Insulin receptors in beta-cells are critical for islet compensatory growth response to insulin resistance. Proc. Natl Acad. Sci. USA 104, 8977-8982 (2007).

60. Yates, A. et al. Ensembl 2016. Nucleic Acids Res. 44, D710-D716 (2016).

61. Roehr, J. T., Dieterich, C. \& Reinert, K. Flexbar 3.0-SIMD and multicore parallelization. Bioinformatics 33, 2941-2942 (2017).

62. Langmead, B. \& Salzberg, S. L. Fast gapped-read alignment with Bowtie 2. Nat. Methods 9, 357-359 (2012)

63. Dobin, A. et al. STAR: ultrafast universal RNA-seq aligner. Bioinformatics 29, 15-21 (2013).

64. Trapnell, C. et al. Transcript assembly and quantification by RNA-Seq reveals unannotated transcripts and isoform switching during cell differentiation. Nat. Biotechnol. 28, 511-515 (2010).

65. Wagle, P., Nikolic, M. \& Frommolt, P. QuickNGS elevates Next-Generation Sequencing data analysis to a new level of automation. BMC Genomics 16, 487 (2015).

66. Trapnell, C., Pachter, L. \& Salzberg, S. L. TopHat: discovering splice junctions with RNA-Seq. Bioinformatics 25, 1105-1111 (2009).

67. Love, M. I., Huber, W. \& Anders, S. Moderated estimation of fold change and dispersion for RNA-seq data with DESeq2. Genome Biol. 15, 550 (2014).

68. Trapnell, C. et al. Differential gene and transcript expression analysis of RNAseq experiments with TopHat and Cufflinks. Nat. Protoc. 7, 562-578 (2012).

69. Madsen, J. G. S. et al. Integrated analysis of motif activity and gene expression changes of transcription factors. Genome Res. 28, 243-255 (2018).

70. Bernstein, B. E. et al. The NIH Roadmap Epigenomics Mapping Consortium. Nat. Biotechnol. 28, 1045-1048 (2010).

71. Haeussler, M. et al. Evaluation of off-target and on-target scoring algorithms and integration into the guide RNA selection tool CRISPOR. Genome Biol. 17, 148 (2016)

72. Troder, S. E. et al. An optimized electroporation approach for efficient CRISPR/Cas9 genome editing in murine zygotes. PloS One 13, e0196891 (2018).

73. Severgnini, M. et al. A rapid two-step method for isolation of functional primary mouse hepatocytes: cell characterization and asialoglycoprotein receptor based assay development. Cytotechnology 64, 187-195 (2012).
74. Poupeau, A. \& Postic, C. Cross-regulation of hepatic glucose metabolism via ChREBP and nuclear receptors. Biochim. Biophys. Acta 1812, 995-1006 (2011).

75. Quinlan, A. R. \& Hall, I. M. BEDTools: a flexible suite of utilities for comparing genomic features. Bioinformatics 26, 841-842 (2010).

\section{Acknowledgements}

We thank Brigitte Hampel and Pia Scholl for H/E stainings, Jens Alber for technical support with indirect calorimetry measurements and mouse handling, and Lisa Czaja for help with RNA-Seq. MPJ was supported by an Albert Renold Travel Fellowship from the European Association for the Study of Diabetes (EASD). H.T. was awarded with a ShortTerm Fellowship from the European Molecular Biology Organization (EMBO). J.W.K., M.O. and E.S. were supported by the Emmy-Noether Program of the Deutsche Forschungsgemeinschaft (DFG, KO4728/1.1). J.W.K., B.D.L. and C.K. received funding from the University of Southern Denmark (SDU) and Danish Diabetes Academy (DDA), which is funded by the Novo Nordisk Foundation (NNF). J.W.K., R.L., E.F.R., M.P.J. and P.K. received support from European Research Council Starting (Grant No. 675014). E.S. was supported by Evangelisches Studienwerk Villigst e.V. S.K. appreciates a PhD stipend from the German Academic Exchange Service (DAAD). N.R.H. was awarded with a PhD stipend from the Cologne Graduate School for Ageing (CGA). R.N.K. and D.F.D. received support from NIH Grants RO1 DK103215, RO1 DK67536, RO1 117639, and DK P036836. J.C.L. is supported by UPLIFT: UCLA Postdocs' Longitudinal Investment in Faculty (Award \# K12 GM106996). L.Y. and H.C. were supported by intramural NIH research funding grants (references 1ZIAHL006103 and 1ZIAHL006159). L.Y. was supported by NIH grant K22HL139921. T.Q. de A.V. is funded by NIH grants DK112119 and HL122677 and P.A.E. and T.Q. de A.V. are funded by DK118064.

\section{Author contributions}

M.P.J., N.R.H., J.C.L., E.S., N.M., R.L., I.D., S.K., M.O., H.T., M.A., H.B., L.Y. and E.F.R. performed the experiments. R.S. performed training in CRISPR/Cas9 editing. M.H., L.S. J.H. and U.K. provided human liver biopsies and information about patient characteristics and helped with data analysis. S.E.T. and B.Z. performed CRISP/Cas9 gene targeting. P.F., P.K., C.D., C.A.K., B.D.L. and I.U. helped with RNA-Seq and ChiP-Seq analysis, data integration, analysis of mouse/human IncRNA synteny, and discussions. H.C. conceived and analyzed LincIRS2 expression after adenoviral TF transduction. D.D.J. analyzed LincIRS2 expression in LIRKO mice. P.A.E. provided discussions and infrastructure. B.Z. and S.T. generated LincIRS2-knockout mice. R.G.L. provided Mafg ASOs. M.Y. provided Maff/Mafk-knockout mice. M.P.J., N.R.H., H.B., M.A., H.C., R.N.K., H.B., I.U., E.F.R., T.Q.A.V. and J.W.K. conceived the experiments.

\section{Competing interests}

The authors declare no competing interests.

\section{Additional information}

Supplementary information is available for this paper at https://doi.org/10.1038/s41467 020-14323-y.

Correspondence and requests for materials should be addressed to T.Q.d.A.V. or J.-W.K.

Peer review information Nature Communications thanks Annette Schürmann, Songlin Wang and other, anonymous, reviewer(s) for their contribution to the peer review of this work.

Reprints and permission information is available at http://www.nature.com/reprints

Publisher's note Springer Nature remains neutral with regard to jurisdictional claims in published maps and institutional affiliations.

Open Access This article is licensed under a Creative Commons Attribution 4.0 International License, which permits use, sharing, adaptation, distribution and reproduction in any medium or format, as long as you give appropriate credit to the original author(s) and the source, provide a link to the Creative Commons license, and indicate if changes were made. The images or other third party material in this article are included in the article's Creative Commons license, unless indicated otherwise in a credit line to the material. If material is not included in the article's Creative Commons license and your intended use is not permitted by statutory regulation or exceeds the permitted use, you will need to obtain permission directly from the copyright holder. To view a copy of this license, visit http://creativecommons.org/ licenses/by/4.0/.

(C) The Author(s) 2020 
Marta Pradas-Juni, 2,3, Nils R. Hansmeier ${ }^{2,3}$, Jenny C. Link (10) ${ }^{4,5}$, Elena Schmidt $\mathbb{D}^{2,3}$, Bjørk Ditlev Larsen ${ }^{1}$, Paul Klemm (i) 2,3, Nicola Meola1, Hande Topel (1) 1,6,7, Rute Loureiro 1,2,3, Ines Dhaouadi ${ }^{2,3}$, Christoph A. Kiefer (1) ${ }^{1}$, Robin Schwarzer ${ }^{3}$, Sajjad Khani ${ }^{2,3}$, Matteo Oliverio (1) ${ }^{2,3}$, Motoharu Awazawa ${ }^{2,8}$, Peter Frommolt ${ }^{3}$, Joerg Heeren (10 ${ }^{9}$, Ludger Scheja ${ }^{9}$, Markus Heine ${ }^{9}$, Christoph Dieterich ${ }^{10}$, Hildegard Büning ${ }^{11}$, Ling Yang (iD) ${ }^{12,19}$, Haiming Cao ${ }^{12}$, Dario F. De Jesus (10 ${ }^{13}$, Rohit N. Kulkarni (1) ${ }^{13}$, Branko Zevnik (1) ${ }^{14}$, Simon E. Tröder (1) ${ }^{14}$, Uwe Knippschild $^{15}$, Peter A. Edwards 4,5 , Richard G. Lee (10 ${ }^{16}$, Masayuki Yamamoto ${ }^{17}$, Igor Ulitsky (10 ${ }^{18}$, Eduardo Fernandez-Rebollo (1,20, Thomas Q. de Aguiar Vallim ${ }^{4,5,20 \star}$ \& Jan-Wilhelm Kornfeld (1) 1,2,3,20*

${ }^{1}$ Functional Genomics and Metabolism Unit, Department for Biochemistry and Molecular Biology, University of Southern Denmark, Campusvej 55, 5230 Odense M, Denmark. ${ }^{2}$ Max Planck Institute for Metabolism Research, Gleueler Strasse 50, 50931 Cologne, Germany. ${ }^{3}$ Cologne Cluster of Excellence-Cellular Stress Responses in Ageing-associated Diseases (CECAD), Medical Faculty, University of Cologne, Joseph-Stelzmann-Str. 26, 50931 Cologne, Germany. ${ }^{4}$ Department of Biological Chemistry, University of California, Los Angeles (UCLA), 650 Charles E. Young Drive South, Los Angeles, CA 90095, USA. 5 Department of Medicine, Division of Cardiology, UCLA, 650 Charles E. Young Drive South, Los Angeles, CA 90095 USA. ${ }^{6}$ Izmir Biomedicine and Genome Center (IBG), Mithatpasa Ave. 58/5, 35340 Izmir, Turkey. ${ }^{7}$ Department of Medical Biology and Genetics, Graduate School of Health Sciences, Dokuz Eylul University, Mithatpasa Ave. 1606, 35330 Izmir, Turkey. ${ }^{8}$ Diabetes Research Center, Research Institute, National Center for Global Health and Medicine, Tokyo 162-8655, Japan. ${ }^{9}$ Department of Biochemistry and Molecular Cell Biology, Martinistraße 52, 20246 Hamburg, Germany. ${ }^{10}$ Section of Bioinformatics and Systems Cardiology, Klaus Tschira Institute for Integrative Computational Cardiology, University Hospital Heidelberg, Im Neuenheimer Feld 669, 69120 Heidelberg, Germany. ${ }^{11}$ Institute of Experimental Hematology, Hanover Medical School, Carl-Neuberg-Str. 1, 30625 Hannover, Germany. ${ }^{12}$ Cardiovascular Branch, National Heart Lung and Blood Institute, Bethesda, MD 20892, USA. ${ }^{13}$ Islet Cell and Regenerative Biology, Joslin Diabetes Center, Department of Medicine, Brigham and Women's Hospital, Harvard Stem Cell Institute, Harvard Medical School, Boston 02215 MA, USA. ${ }^{14}$ CECAD in vivo Research Facility, Medical Faculty, University of Cologne, Joseph-Stelzmann-Str. 26, 50931 Cologne, Germany. ${ }^{15}$ Department of General and Visceral Surgery, University Hospital Ulm, Albert-Einstein Allee 93, 89081 Ulm, Germany. ${ }^{16}$ IONIS Pharmaceuticals, Carlsbad, CA 92010, USA. ${ }^{17}$ Department of Medical Biochemistry, Tohoku Medical Megabank Organization, Sendai 980-8573, Japan. ${ }^{18}$ Department of Biological Regulation, Weizmann Institute of Science, Rehovot 76100, Israel. ${ }^{19}$ Present address: Lewis Katz School of Medicine, Temple University, Philadelphia, PA 19140, USA. ${ }^{20}$ These authors jointly supervised this work: Eduardo Fernandez-Rebollo, Thomas Q. de Aguiar Vallim, Jan-Wilhelm Kornfeld. *email: TVallim@mednet.ucla.edu; janwilhelmkornfeld@bmb.sdu.dk 